DAMTP-2000-80

hep-th/0008125

\title{
Derivative corrections to D-brane actions with constant background fields
}

\author{
Niclas Wyllard円 \\ Department of Applied Mathematics and Theoretical Physics, \\ Centre for Mathematical Sciences, University of Cambridge, \\ Wilberforce Road, Cambridge, CB3 0WA, United Kingdom
}

\begin{abstract}
We study derivative corrections to the effective action for a single D-brane in type II superstring theory coupled to constant background fields. In particular, within this setting we determine the complete expression for the (disk-level) fourderivative corrections to the Born-Infeld part of the action. We also determine $2 n$ form $2 n$-derivative corrections to the Wess-Zumino term. Both types of corrections involve all orders of the gauge field strength, $F$. The results are obtained via string $\sigma$-model loop calculations using the boundary state operator language. The corrections can be succinctly written in terms of the Riemann tensor for a nonsymmetric metric.
\end{abstract}

\section{Introduction}

Most work on D-brane effective actions have focused on the lowest order terms in a derivative expansion. For the case of a single brane in type II superstring theory, these terms have been completely determined and are well known. They are of two types: the parity-violating Wess-Zumino term [1], which includes couplings to the Ramond-Ramond background fields, and the parity-conserving Born-Infeld part [2 《4. The inclusion of fermions to form a $\kappa$-symmetric action (including the couplings to a general supergravity background) is known [5]. It has also been shown (for the lowest order terms) that Tduality is realised in the D-brane effective action [6, 7]. The lowest order action is in fact highly constrained and can be completely determined in a variety of ways. Apart from explicit calculations [2 1 , 8], these terms can also be fixed completely using the constraints of supersymmetry in the guise of $\kappa$-symmetry [9], and also (for the Born-Infeld part) by Lorentz covariance combined with T-duality [10] or via the connection to its noncommutative version [11]. The requirement of electromagnetic Montonen-Olive duality

\footnotetext{
${ }^{1}$ N.Wyllard@damtp.cam.ac.uk
} 
(or more generally $\mathrm{SL}(2, \mathbb{Z})$ invariance) of the D3-brane also puts strong constraints on the form of the action 12, 13. Together with certain conditions on the solutions it uniquely leads to the Born-Infeld action [14. In addition, the Wess-Zumino part can be determined by anomaly cancellation arguments 15.

There are in general derivative corrections to the lowest order terms, which, because of the fundamental role played by the D-branes (see [16] for reviews), are presumably important and may possibly lead to a deeper understanding of the properties of D-branes and various dualities. The derivative corrections fall into two classes. Firstly, there are corrections involving derivatives of the world-volume fields, i.e the gauge field $F_{\mu \nu}$ and the embedding fields $\partial_{\mu} X^{i}$, where $X^{i}$ are the transverse scalars 2 . Secondly, there are corrections which involve the pullback of expressions containing derivatives of the bulk fields $\left(g_{M N}, B_{M N}, \Phi\right.$ and the $C_{p}$ 's) to the world volume of the brane. In general there are of course also combinations of the two types. Corrections of the second type were discussed in [17], where $\alpha^{2}$ corrections to the Born-Infeld part arising from the gravity sector of the superstring background were determined by comparison with earlier string calculations [8, 18. These corrections involve quadratic expressions of the pullbacks of the bulk Riemann tensor to the normal and tangent bundles. Corrections to the Wess-Zumino term involving the bulk Riemann tensor were determined using anomaly cancellation arguments in [15] and have subsequently also been obtained via direct string theory calculations [19]. In [17] corrections involving the second fundamental form of the embedding were also determined (both for the Wess-Zumino and Born-Infeld parts of the action). Since the second fundamental form is constructed out of derivatives of the embedding fields, certain corrections of the first type were thus obtained.

In this paper we study derivative corrections to the gauge sector of the effective action of a single D-brane in type II superstring theory. (We only consider branes which preserve half of the supersymmetries and not the recently much studied "non-BPS" D-branes. We also do not consider the non-abelian case.) There are several different methods available for calculating such corrections. Firstly, there is the string S-matrix method [20], where one proceeds by directly calculating string scattering amplitudes and from the result reconstruct the effective action. String S-matrix techniques are powerful, but for the Dbranes they have the drawback that they are perturbative in powers of the gauge field strength $F$; fortunately, there are other methods available which are non-perturbative in powers of the gauge field $F$, i.e. they are powerful enough to allow one to sum the entire perturbative series. One of these methods is the so called $\beta$-function method, where one calculates the $\beta$-function of the string $\sigma$-model as a loop expansion and interpret its vanishing as equations of motion for the world-volume fields. This approach is closely related to the requirement of conformal invariance. Using this method the Born-Infeld part of the lowest order action was obtained for the ten-dimensional case (or in modern language, for the D9-brane) in [3] and for the lower-dimensional D-branes in [4]. A method

\footnotetext{
${ }^{2}$ We will throughout this paper consider $F_{\mu \nu}$ and $\partial_{\mu} X^{i}$ to be of zeroth order. When we speak of derivative corrections we thus mean corrections which vanish when $F$ and $\partial X$ are constant.

${ }^{3}$ We absorb a factor of $2 \pi \alpha^{\prime}$ into $F$ and $\partial X$ to make them dimensionless; $\alpha^{\prime 2}$ corrections thus involve four derivatives.
} 
in the same vein is the partition function method [2, 21, 22]. This was the method used when the parity-conserving part of the effective action was first obtained for the ninebrane case in [2] and has subsequently been extended to the lower-dimensional cases as well [13]. For both the superstring and the bosonic string this method (for the lowest order term, i.e. neglecting derivatives) leads to the well known Born-Infeld determinant form. When derivative corrections are included the method gives the wrong result for the case of the bosonic string [22]. For the superstring case, however, it has been shown that the method gives correct results [22]. Closely related to the partition function method is the boundary state formalism 23 25] (see [26] for a recent review). The boundary state is an important tool in the study of D-branes in type II superstring theory and encodes various properties of the D-branes, including the effective action. The boundary state formalism has the advantage that it leads to a natural way of determining the WessZumino term, viz. by calculating the overlap of the state representing the RR form fields with the boundary state. The extension of the boundary state method to incorporate a non-constant gauge field was recently discussed in detail [27, 28] (see also [24]).

For the D-branes in type II superstring theory the $\mathcal{O}\left(\alpha^{\prime}\right)$ corrections involving the gauge field $F$ to the Born-Infeld part of the action are known to vanish 22, 28]. The $\mathcal{O}\left(\alpha^{\prime}\right)$ corrections to the Wess-Zumino term also vanish (see e.g. [2\&]). Thus, the first non-trivial corrections appear at order $\alpha^{\prime 2}$. The $\alpha^{\prime 2}$ corrections to the Born-Infeld term involving four $F$ 's (and four derivatives) are known [22 from comparison with earlier string amplitude calculations (see e.g. [29]). In this paper we determine the complete expression for the $\alpha^{2}$ corrections to the Born-Infeld part of a D9-brane coupled to constant background fields at disk level in the string coupling-constant perturbation expansion. We also determine $2 n$ form $2 n$-derivative $\left(\alpha^{\prime n}\right)$ corrections to the Wess-Zumino part. By T-duality (dimensional reduction), all lower-dimensional cases can be obtained although we do not study this issue in detail.

In the next section we review some results in the literature for future reference and also introduce our notation. In section 3 we calculate $2 n$-form $2 n$-derivative corrections to the Wess-Zumino term and in section 4 we calculate the $\alpha^{2}$ corrections to the BornInfeld action and also show that the four- $F$ part correctly reproduces the previously known result. In section 5 we then move on to relate our results to what is known in the literature about the lower-dimensional cases. In particular, we show that by dimensional reduction of the Wess-Zumino term to $p+1$ dimensions, the part involving only $\partial X$ (i.e. setting $F$ in the lower dimension to zero) correctly reproduces the corrections to the Wess-Zumino term involving the second fundamental form. In the final section we make the observation that the four-index tensor in terms of which the corrections determined in sections 3 and 1 can be expressed is reminiscent of the usual expression for the Riemann tensor. We make this idea precise by showing that the tensor can be identified with the Riemann tensor for the non-symmetric metric $h=\delta+F$, where $\delta$ is the flat metric. In the appendices we list our conventions and give some technical details of the calculations. 


\section{Preliminaries}

In this section we will review some known facts to introduce our approach and some terminology. We will focus on the case of the D9-brane in the type IIB theory. Comments on the lower-dimensional cases will appear in a later section. The boundary conditions for an open string ending on a D9-brane are: $P_{\mu}=0$ and, for the fermionic coordinates, $\bar{\Psi}^{\mu}=0$ (see appendix A for a description of our conventions). The boundary state $|B\rangle$ is a BRST-invariant state which imposes these boundary conditions, i.e. it satisfies $P_{\mu}|B\rangle=0$ and $\bar{\Psi}^{\mu}|B\rangle=0$. In the presence of a background gauge field, $A_{\mu}(X)$, with field strength $F_{\mu \nu}(X)=\frac{\partial}{\partial X^{\mu}} A_{\nu}(X)-\frac{\partial}{\partial X^{\nu}} A_{\mu}(X)$, the boundary condition requirements on the boundary state $|B(F(X))\rangle$ are replaced by [24]

$$
\begin{array}{r}
\left(2 \pi \alpha^{\prime} P_{\mu}+F_{\mu \nu}(X) \partial_{\sigma} X^{\nu}\right)|B(F(X))\rangle=0 \\
\left(\bar{\Psi}_{\mu}-F_{\mu \nu}(X) \Psi^{\nu}\right)|B(F(X))\rangle=0 .
\end{array}
$$

When restricting to the zero-mode part of $F(X)$, i.e. $F(x)$, these conditions can be written in terms of oscillators as

$$
\begin{aligned}
{\left[(1-F)_{\mu \nu} \alpha_{n}^{\nu}+(1+F)_{\mu \nu} \tilde{\alpha}_{-n}^{\nu}\right]|B(F)\rangle } & =0, \\
{\left[(1-F)_{\mu \nu} d_{n}^{\nu}-i \eta(1+F)_{\mu \nu} \tilde{d}_{-n}^{\nu}\right]|B(F)\rangle_{\mathrm{R}} } & =0, \\
{\left[(1-F)_{\mu \nu} b_{r}^{\nu}-i \eta(1+F)_{\mu \nu} \tilde{b}_{-r}^{\nu}\right]|B(F)\rangle_{\mathrm{NS}} } & =0 .
\end{aligned}
$$

Here we have introduced the notation $|B(F)\rangle:=\mid B(F(x)\rangle$. In (2.2) $\eta \in\{+,-\}$ is the spin structure and the subscripts R and NS refer to the Ramond and Neveu-Schwarz sectors, respectively.

A formal BRST-invariant solution to the equations (2.1) is 27, 28

$$
|B(F(X))\rangle=e^{-\frac{i}{2 \pi \alpha^{\prime}} \int d \sigma\left[\partial_{\sigma} X^{\mu} A_{\mu}(X)-\frac{1}{2} \Psi^{\mu} \Psi^{\nu} F_{\mu \nu}(X)\right]}|B\rangle .
$$

This expression can be succinctly written in terms of the superfield $\phi^{\mu}=X^{\mu}+\theta \Psi^{\mu}$ and the super-covariant derivative $D=\theta \partial_{\sigma}-\partial_{\theta}$, as $|B(F(X))\rangle=e^{-\frac{i}{2 \pi \alpha^{\prime}} \int d \sigma \mathrm{d} \theta D \phi^{\mu} A_{\mu}(\phi)}|B\rangle$. As will become clear later, we will not need the explicit form of $|B\rangle$ in this paper; the properties (2.1), (2.2) are sufficient for our purposes.

It is known [24 that (in the absence of the NSNS B-field) the parity-conserving ("BornInfeld") contribution (including derivative corrections) to the effective action is proportional to $\langle 0 \mid B(F(X))\rangle_{\mathrm{NS}}$, i.e.

$$
S_{\mathrm{BI}} \propto\left\langle 0\left|e^{-\frac{i}{2 \pi \alpha^{\prime}} \int \mathrm{d} \sigma \mathrm{d} \theta D \phi^{\mu} A_{\mu}(\phi)}\right| B\right\rangle_{\mathrm{NS}}
$$

This expression is the operator analogue of the path integral expression in the partition function approach:

$$
S_{\mathrm{BI}} \propto \int \mathrm{d}[X] \mathrm{d}[\Psi] e^{-\frac{i}{2 \pi \alpha^{\prime}} \int \mathrm{d} \sigma \mathrm{d} \theta D \phi^{\mu} A_{\mu}(\phi)} .
$$

\footnotetext{
${ }^{4}$ Here we have absorbed a factor of $2 \pi \alpha^{\prime}$ into $A_{\mu}$; what we call $F$ is thus really $2 \pi \alpha^{\prime} F$.
} 
As indicated by the notation, the part of the effective action obtained from (2.4) receives contributions only from the NS sector as we will see below. The coupling to a constant $B$-field can be obtained by observing that the only effect of the addition of a constant $B$-field to the string $\sigma$-model action is to shift $F$ to $F+B$. Another method to determine the (linear) couplings to the background fields is to calculate the overlap of the boundary state with the state corresponding to the relevant background field. For the case of a constant $B$-field the result of the two approaches will be the same. The latter method is, however, more complicated for the cases treated in this paper. The dependence on a constant dilaton, $\Phi$, can also easily be determined: the only effect (in the string frame) will be an overall multiplicative factor $e^{-\Phi}$, which can be understood from the fact that we discuss disk-level contributions in the string coupling perturbation expansion.

It is known that the parity-violating part of the effective action involving the linear couplings to the background $\mathrm{RR}$ form fields is obtained by calculating the overlap between the state, $|C\rangle$, representing these form fields, and the boundary state in the $\mathrm{R}$ sector (see e.g. [30]), i.e.

$$
S_{\mathrm{WZ}} \propto\langle C| B(F(X)\rangle_{\mathrm{R}} .
$$

When neglecting derivatives acting on $F_{\mu \nu}$, we can replace $\int \mathrm{d} \sigma \mathrm{d} \theta D \phi^{\mu} A_{\mu}(\phi)$ with $-\frac{1}{2} \int \mathrm{d} \sigma \mathrm{d} \theta D \phi^{\mu} \phi^{\nu} F_{\mu \nu}$. The lowest order contribution to the parity-conserving part of the effective action is thus

$$
S_{\mathrm{BI}}^{(0)} \propto\langle 0 \mid B(F)\rangle_{\mathrm{NS}}, \text { where }|B(F)\rangle_{\mathrm{NS}}:=e^{\frac{i}{4 \pi \alpha^{\prime}} \int \mathrm{d} \sigma \mathrm{d} \theta D \phi^{\mu} \phi^{\nu} F_{\mu \nu}}|B\rangle_{\mathrm{NS}} .
$$

A simple way to determine $S_{\mathrm{BI}}^{(0)}$ is to differentiate (2.7) with respect to $F_{\mu \nu}$. This will bring down a factor $\frac{i}{4 \pi \alpha^{\prime}} \int \mathrm{d} \sigma \mathrm{d} \theta D \phi^{\mu} \phi^{\nu}$ from the exponent. In this expression, only the non-zero-mode part of the $\phi^{\prime}$ 's, $\tilde{\phi}^{\eta}:=\phi^{\eta}-x^{\eta}$, contributes. The next step is to use the properties of $|B(F)\rangle(2.2)$ to turn all oscillators in $\tilde{\phi}^{\nu}$ into creation operators; we will denote the resulting expression $\tilde{\phi}_{+}^{\nu}$. Finally, one moves $\tilde{\phi}_{+}^{\nu}$ past $D \tilde{\phi}^{\mu}$ and annihilates it against $\langle 0|$ and in the process pick up a commutator $\left[D \tilde{\phi}^{\mu}\left(\sigma_{1}, \theta_{1}\right), \tilde{\phi}_{+}^{\nu}\left(\sigma_{2}, \theta_{2}\right)\right]_{1 \rightarrow 2}$. We introduce the notation

$$
G_{12}^{\lambda \mu}:=\left[\tilde{\phi}^{\lambda}\left(\sigma_{1}, \theta_{1}\right), \tilde{\phi}_{+}^{\mu}\left(\sigma_{2}, \theta_{2}\right)\right]=D_{12}^{\lambda \mu}-\theta_{1} \theta_{2} K_{12}^{\lambda \mu} .
$$

In components, $D_{12}^{\mu \nu}=\left[\tilde{X}^{\lambda}\left(\sigma_{1}\right), \tilde{X}_{+}^{\mu}\left(\sigma_{2}\right)\right]$ and $K_{12}^{\mu \nu}=\left\{\tilde{\Psi}^{\mu}, \tilde{\Psi}_{+}^{\nu}\right\}$. An explicit calculation leads to divergent expressions which need to be regularised. In this paper we will use the following regularisation

$$
\begin{aligned}
D_{12}^{\mu \nu} & =2 \alpha^{\prime} \sum_{n=1}^{\infty}\left[\frac{e^{-\epsilon n}}{n}\left(h_{S}^{\mu \nu} \cos \left[n\left(\sigma_{2}-\sigma_{1}\right)\right]+i h_{A}^{\mu \nu} \sin \left[n\left(\sigma_{2}-\sigma_{1}\right)\right]\right)\right] \\
& =\alpha^{\prime} \sum_{n=1}^{\infty}\left[\frac{e^{-\epsilon n}}{n}\left(h^{\mu \nu} e^{i n\left(\sigma_{2}-\sigma_{1}\right)}+h^{\nu \mu} e^{-i n\left(\sigma_{2}-\sigma_{1}\right)}\right)\right] \\
K_{12}^{\mu \nu} & =2 \alpha^{\prime} \sum_{r>0}\left[e^{-\epsilon r}\left(-h_{S}^{\mu \nu} \sin \left[r\left(\sigma_{2}-\sigma_{1}\right)\right]+i h_{A}^{\mu \nu} \cos \left[r\left(\sigma_{2}-\sigma_{1}\right)\right]\right)\right] \\
& =i \alpha^{\prime} \sum_{r>0}^{\infty}\left[e^{-\epsilon r}\left(h^{\mu \nu} e^{i r\left(\sigma_{2}-\sigma_{1}\right)}-h^{\nu \mu} e^{-i r\left(\sigma_{2}-\sigma_{1}\right)}\right)\right]
\end{aligned}
$$


where $\epsilon>0$ is the regulator. In (2.9) we have used the notation $h^{\mu \nu}=\left(\frac{1}{1+F}\right)^{\mu \nu}$ for the inverse of $h_{\mu \nu}:=(1+F)_{\rho \nu}$, (i.e., $h^{\mu \nu} h_{\nu \rho}=\delta_{\rho}^{\mu}$ and $h_{\rho \nu} h^{\nu \mu}=\delta_{\rho}^{\mu}$ ), and also $h_{A}^{\mu \nu}=\frac{1}{2}\left[h^{\mu \nu}-h^{\nu \mu}\right]$ and $h_{S}^{\mu \nu}=\frac{1}{2}\left(h^{\mu \nu}+h^{\nu \mu}\right)$. In the expression for $K_{12}^{\mu \nu}, r$ runs over the (positive) half integers in the NS sector, whereas it takes (positive) integer values in the $\mathrm{R}$ sector. The results (2.9) agree with the usual expressions for the $F$-dependent propagators on the boundary of the disk [3,22]. The spin structure parameter $\eta$ drops out of the propagator, so the GSO projection (sum over spin structures) can be absorbed into the zeroth order boundary state and will not enter in our discussion.

We now return to the determination of $S_{\mathrm{BI}}^{(0)}$. From the above results it follows that $\int \mathrm{d} \theta_{1}\left[D \phi^{\mu}\left(\sigma_{1}, \theta_{1}\right), \phi_{+}^{\nu}\left(\sigma_{2}, \theta_{2}\right)\right]_{1 \rightarrow 2}=-2 i \alpha^{\prime} h_{A}^{\mu \nu}\left[\sum_{n} e^{-\epsilon n}-\sum_{r} e^{-\epsilon r}\right]$, which when the regulator is removed becomes $i \alpha^{\prime} h_{A}^{\mu \nu}$. From the integration over $\sigma$ we get an additional factor of $2 \pi$. Thus, we obtain the following differential equation: $\frac{\partial S_{\mathrm{BI}}^{(0)}}{\partial F_{\mu \nu}}=-\frac{1}{2} h_{A}^{\mu \nu} S_{\mathrm{BI}}^{(0)}$, for the lowest order term, which (up to a multiplicative constant, which is identified with minus the tension, $T_{9}$ ) has the usual Born-Infeld action

$$
S_{\mathrm{BI}}^{(0)}=-T_{9} \int \mathrm{d}^{10} x \sqrt{\operatorname{det}(1+F)}=-T_{9} \int \mathrm{d}^{10} x \sqrt{\operatorname{det} h},
$$

as its unique solution. This is most easily proven by going to the special Lorentz frame where $F_{\mu \nu}$ is block diagonal.

The lowest order contribution to the Wess-Zumino term was calculated in detail in [30] using the present framework. Only the zero-mode part of $|B(F)\rangle$ contributes (since $\left[D_{1} G_{12}^{\mu \nu}\right]_{1 \rightarrow 2}=0$ in the $\mathrm{R}$ sector) and the result is the well known expression

$$
S_{\mathrm{WZ}}^{(0)}=T_{p} \int C \wedge e^{F}
$$

where $C$ is the sum of the RR potential forms.

To study derivative corrections to the effective action we will expand $A_{\mu}(\phi)$ around the zero mode of $\phi$, i.e. we split $\phi$ as $\varphi_{0}+\tilde{\phi}$, and Taylor expand:

$$
A_{\mu}(\phi)=\sum_{n=0}^{\infty} \frac{1}{n !} \tilde{\phi}^{\nu_{1}} \cdots \tilde{\phi}^{\nu_{n}} \partial_{\nu_{1}} \cdots \partial_{\nu_{n}} A_{\mu}\left(\varphi_{0}\right)
$$

This procedure leads to a well defined perturbative expansion in terms of the number of derivatives. Inserting the expansion (2.12) into $\int \mathrm{d} \sigma \mathrm{d} \theta D_{\sigma} \phi^{\mu} A_{\mu}(\phi)$ results in

$$
\begin{aligned}
& -\int \mathrm{d} \sigma \mathrm{d} \theta \sum_{k=0}^{\infty} \frac{1}{(k+1) !} \frac{k+1}{k+2} D \tilde{\phi}^{\nu} \tilde{\phi}^{\mu} \tilde{\phi}^{\lambda_{1}} \cdots \tilde{\phi}^{\lambda_{k}} \partial_{\lambda_{1}} \cdots \partial_{\lambda_{k}} F_{\mu \nu}(x) \\
& -\int \mathrm{d} \sigma\left[\tilde{\Psi}^{\mu} \psi_{0}^{\nu}+\frac{1}{2} \psi_{0}^{\mu} \psi_{0}^{\nu}\right] \sum_{k=0}^{\infty} \frac{1}{k !} \tilde{X}^{\lambda_{1}} \cdots \tilde{X}^{\lambda_{k}} \partial_{\lambda_{1}} \cdots \partial_{\lambda_{k}} F_{\mu \nu}(x) .
\end{aligned}
$$

In the NS sector $\varphi_{0}^{\mu}=x^{\mu}$, whereas in the $\mathrm{R}$ sector $\varphi_{0}^{\mu}=x^{\mu}+\theta \psi_{0}$. Hence, in the NS sector the second term in (2.13) is absent. The $k=0$ terms in the above expansion give 
rise to the $F(x)$ dependence in $|B(F)\rangle$. As discussed in [27, 28] the boundary state (2.3) is formal in the sense that the perturbative expansion it generates contains divergences which have to be regularised. There are two ways to view these divergencies. Either one takes the view that the $F$ one started with, i.e. the one appearing in the boundary state, is the "true" $F$. The terms multiplying the divergent terms then have to vanish. These conditions are closely related to the vanishing of the $\beta$-function in the $\beta$-function approach as discussed in [27, 28]. The other way to view the divergencies is to say that one started with the wrong $F$, and to take this fact into account by making field redefinitions to absorb the divergencies (since the theory is renormalisable this can always be done). We will take the latter viewpoint in this paper. The effective D-brane action is thus obtained from (2.4) and (2.6) after renormalisation. At least for the parity-conserving part one can also reformulate the algebraic operator approach used so far in terms of Feynman diagrams. Since $F$ satisfies the Bianchi identity, $\partial_{[\lambda} F_{\mu \nu]}=0$, there will be relations between different contributions which reduce the rather large number of diagrams and simplifies the analysis. We found it easier to take such relations into account in the operator language.

We will now briefly review, using the boundary state formalism, what is known about the $\mathcal{O}\left(\alpha^{\prime}\right)$ corrections to the effective action for a D-brane or, stated in another way, the corrections involving two additional derivatives compared to the zeroth order terms (2.10), (2.11). To this end we only need to keep the first three terms $(k=0,1,2)$ in the expansion (2.13), insert them into the exponential in (2.3) and expand (again retaining only the terms with at most two derivatives). We only need to consider terms with an even number of derivatives, since it is not possible to form a scalar out of $F_{\mu \nu}$ and an odd number of derivatives.

Let us start by discussing the Wess-Zumino part. The two-derivative corrections to the Wess-Zumino term are given by $\langle C| B(F(X)\rangle_{\mathrm{R}}$, with $\mid B(F(X)\rangle_{\mathrm{R}}$ expanded to second order in derivatives. In the various correlation functions that arise we let, as before, the $\tilde{\phi}^{\mu}$ to the far right act on the boundary state $|B(F)\rangle$ to turn annihilation operators into creation operators using the property (2.1) and then move it to the left and finally annihilate it against $\langle C|$. In the process we will pick up commutators of the form (2.8). Continuing this process gives an expression for the contribution to the action expressed in terms of the propagator, $G$. The corrections can be classified according to how many zero modes the expressions from which they arise contains (which, as will be clear later, in turn translates into forms of different degrees). We will now show that the part involving no zero modes vanishes to any order in the derivative perturbation theory. More precisely, we will show that

$$
\left\langle C\left|\left(\int \mathrm{d} \theta_{1} D_{1} \tilde{\phi} \tilde{\phi}^{k_{1}}\right)\left(\int \mathrm{d} \theta_{2} D_{2} \tilde{\phi} \tilde{\phi}^{k_{2}}\right) \cdots\left(\int \mathrm{d} \theta_{n} D_{n} \tilde{\phi} \tilde{\phi}^{k_{n}}\right)\right| B\right\rangle=0,
$$

where we have suppressed all indices. We start by considering $D_{1} \tilde{\phi}$. We get zero if this term is contracted with a $\tilde{\phi}$ within the same factor since $\left[D_{1} G_{12}\right]_{2 \rightarrow 1}=0$ (which follows from the fact that in the $\mathrm{R}$ sector $\left.K_{12}^{\lambda \mu}=\partial_{2} D_{12}^{\lambda \mu}=-\partial_{1} D_{12}^{\lambda \mu}\right)$. Assume therefore that $D_{1} \tilde{\phi}$ is contracted with a $\tilde{\phi}$ in the $m$ th factor (by integration by parts we can always arrange 
it to be one of the $\tilde{\phi}$ 's rather than $\left.D_{m} \tilde{\phi}\right)$. Now consider $D_{m} \tilde{\phi}$. As before we can not contract it with a $\tilde{\phi}$ within the same factor. Also, if it is contracted with a $\tilde{\phi}$ in the first factor we get zero since $D_{1} G_{1 m} D_{m} G_{1 m} \propto\left(\theta_{1}-\theta_{m}\right)^{2}=0$. Thus we can assume that $D_{m} \tilde{\phi}$ is contracted with a $\tilde{\phi}$ in the $n$th factor. Now look at $D_{n} \tilde{\phi}$. There are two possibilities. 1 ) it is contracted with a $\tilde{\phi}$ in the first factor. In this case we select a $D_{k} \phi$ from another factor and start over. 2) it is contracted with the $r$ th term. In this case we continue as before. It should be clear that the procedure outlined above leads to a result proportional to $\prod_{p, q} D_{p} G_{p q} \propto \prod_{p, q}\left(\theta_{p}-\theta_{q}\right)$, for certain $p, q$. Since there are $j$ factors this has in turn to be proportional to $\prod_{i=1}^{j} \theta_{i}$, but since $\prod_{p, q}\left(\theta_{p}-\theta_{q}\right)$ is translational invariant whereas $\prod_{i=1}^{j} \theta_{i}$ is not, the proportionality constant has to be zero. We thus conclude that there are no derivative corrections to the "zero-form" part of the Wess-Zumino term. By the same argument there can arise no scalar functions (constructed out of $h^{\mu \nu}$ 's and $\partial^{k} F^{\prime}$ s) multiplying the corrections with form degrees $>0$.

Let us now return to the discussion of the $\mathcal{O}\left(\alpha^{\prime}\right)$ corrections to the Wess-Zumino term. The two contributions to the $\left(\psi_{0}\right)^{2}$ part can be removed by the field redefinition 28

$$
\delta F_{\mu \nu}=\alpha^{\prime}\left(\ln \epsilon h^{\lambda \rho} \partial_{\lambda} \partial_{\rho} F_{\mu \nu}-2 \ln (2 \epsilon) h^{(\rho \mid \eta} \partial_{[\mu} F_{\mid \eta \delta} h^{\delta \mid \lambda)} \partial_{\lambda} F_{\rho \mid \nu]}\right)
$$

The $\ln \epsilon$ terms can further be absorbed into a field redefinition of $A_{\mu}$ as

$$
\delta A_{\mu}=\alpha^{\prime} \ln \epsilon h_{S}^{\lambda \rho} \partial_{\lambda} F_{\rho \nu}
$$

but the remaining $\ln 2$ term cannot be absorbed into a redefinition of $A_{\mu}$. The easiest way to see this is to note that the corresponding $\delta F_{\mu \nu}$ is not closed, i.e. $\mathrm{d} \delta F_{\mu \nu} \neq 0$. The $\ln 2$ term is however renormalisation scheme dependent and there exists schemes in which it vanishes. One example of such a scheme is the one discussed in [22]. In this scheme the propagator is replaced by

$$
\begin{aligned}
D_{12}^{\mu \nu} & =2 \alpha^{\prime} \sum_{n=1}^{\infty}\left[\frac{e^{-\epsilon n}}{n}\left(h_{S}^{\mu \nu}\left(F_{n}\right) \cos \left[n\left(\sigma_{2}-\sigma_{1}\right)\right]+i h_{A}^{\mu \nu}\left(F_{n}\right) \sin \left[n\left(\sigma_{2}-\sigma_{1}\right)\right]\right)\right] \\
& =2 \alpha^{\prime} \sum_{n=1}^{\infty}\left[\frac{e^{-\epsilon n}}{n}\left(h^{\mu \nu}\left(F_{n}\right) e^{i n\left(\sigma_{2}-\sigma_{1}\right)}+h^{\nu \mu}\left(F_{n}\right) e^{-i n\left(\sigma_{2}-\sigma_{1}\right)}\right)\right]
\end{aligned}
$$

where $\left(F_{n}\right)_{\mu \nu}:=e^{-\epsilon n} F_{\mu \nu}$. The expression for $K^{\mu \nu}$ is similar. Another scheme is $\zeta$ function regularisation in which both $\ln \epsilon$ and $\ln (2 \epsilon)$ get replaced by $\zeta(1)$ [28. The $\zeta$-function regularisation method becomes less useful at higher loops as the sums become more complicated; we will therefore not use it in this paper.

The $\mathcal{O}\left(\alpha^{\prime}\right)$ corrections to the Born-Infeld part of the action is obtained from (2.4) with (2.3) expanded to second order in derivatives. The calculations can be found in [22] (see also [28]). The resulting expression can be completely removed by the same field redefinition, (2.15), as for the Wess-Zumino term. 


\section{Corrections to the Wess-Zumino term}

In this section we will study derivative corrections involving the world-volume gauge field to the Wess-Zumino term. More precisely, we will determine corrections which couple linearly to the Ramond-Ramond fields.

Let us first note that we need an even number of fermionic zero modes (or equivalently an even number of $\tilde{\Psi}^{\prime} s$ ) to get a non-vanishing result. As is explained in appendix B, this translates into the statement that only corrections of even form degrees are present. Furthermore, the contribution from the zero-mode independent part vanishes (to all orders in the derivative expansion) as a consequence of the general result given in the previous section, thus there are no zero-form corrections.

Let us start by looking at the $\alpha^{\prime 2}$ corrections. We will first discuss the $\left(\psi_{0}\right)^{2}$ terms. These terms can potentially be removed by a field redefinition $\delta F_{\mu \nu}$ of order $\alpha^{2}$, since the zeroth order term transforms as $\delta C e^{F}=C e^{F} \delta F$, which, as explained in appendix $\mathrm{B}$, is of the same form as the corrections arising from the $\left(\psi_{0}\right)^{2}$ terms. Indeed, this is precisely what happens (see appendix B for details). An independent argument showing that it has to happen is the following: the zeroth order Wess-Zumino term satisfies the Bianchi identity $\mathrm{d}\left[C e^{F}\right]=R e^{F}$, where (when $B=$ constant) $R=\mathrm{d} C$. In order for the WessZumino term to still satisfy a Bianchi identity in the presence of two-form corrections, $C e^{F} W_{2}$, the two-form $W_{2}$ has to be closed. This means that the two-form correction can be removed by choosing $\delta F=-W_{2}$, which, since $W_{2}$ is closed, translates (at least locally) into $\delta A=-w_{1}$, where $\mathrm{d} w_{1}=W_{2}$, thus showing that $W_{2}$ can be removed by a field redefinition of $A_{\mu}$. This argument works at any order, showing that the two-form corrections can be removed by a field redefinition. This singles out a particular action, and is a natural way to "gauge" fix the field redefinition ambiguity.

The divergent $\left(\psi_{0}\right)^{4}$ terms at order $\alpha^{\prime 2}$ must be cancelled by the terms arising from inserting $\delta F_{\mu \nu}^{(1)}$ given in (2.15) into the zeroth order action and expanding to second order. That this is works out correctly is a non-trivial check. The remaining finite terms give rise to corrections that can be expressed in terms of the tensor

$$
S_{\rho_{1} \rho_{2} \mu_{1} \mu_{2}}:=\partial_{\rho_{1}} \partial_{\rho_{2}} F_{\mu_{1} \mu_{2}}+2 h^{\nu_{1} \nu_{2}} \partial_{\rho_{1}} F_{\left[\mu_{1} \mid \nu_{1}\right.} \partial_{\rho_{2} \mid} F_{\left.\mu_{2}\right] \nu_{2}} .
$$

Since $S_{\rho_{1} \rho_{2} \mu_{1} \mu_{2}}$ is antisymmetric in its last two indices it can be considered as a differential two-form with two additional indices which we will denote $S_{\rho_{1} \rho_{2}}:=\frac{1}{2 !} S_{\rho_{1} \rho_{2} \mu_{1} \mu_{2}} \mathrm{~d} x^{\mu_{1}} \wedge \mathrm{d} x^{\mu_{2}}$. With this definition, the action including the $\alpha^{2}$ corrections can be written (see appendix B for details)

$$
S_{\mathrm{WZ}}^{(2)}=T_{9} \int C \wedge e^{F} \wedge\left(1+\frac{\left(2 \pi \alpha^{\prime}\right)^{2}}{48} h^{\rho_{4} \rho_{1}} h^{\rho_{2} \rho_{3}} \mathrm{~S}_{\rho_{1} \rho_{2}} \wedge \mathrm{S}_{\rho_{3} \rho_{4}}\right)
$$

One can show that the correction satisfies $\mathrm{d}\left(h^{\rho_{4} \rho_{1}} h^{\rho_{2} \rho_{3}} S_{\rho_{1} \rho_{2}} \wedge S_{\rho_{3} \rho_{4}}\right)=0$, i.e. it is closed, which as discussed above is important for consistency.

It is possible to generalise the 4-form 4-derivative corrections determined above and calculate the corresponding $2 n$-form $2 n$-derivative corrections to the Wess-Zumino term. 
Details can be found in appendix $\mathrm{B}$ and the result is

$$
S=T_{9} \int C \wedge e^{F} \wedge\left(1+\sum_{k=2}^{5} W_{2 k}\right)
$$

where (wedge products are suppressed)

$$
\begin{aligned}
W_{4}= & \left(\alpha^{\prime}\right)^{2} \frac{\zeta(2)}{2} h^{\rho_{4} \rho_{1}} h^{\rho_{2} \rho_{3}} \mathrm{~S}_{\rho_{1} \rho_{2}} \mathrm{~S}_{\rho_{3} \rho_{4}} \\
W_{6}= & \left(\alpha^{\prime}\right)^{3} \frac{\zeta(3)}{3} h^{\rho_{6} \rho_{3}} h^{\rho_{4} \rho_{1}} h^{\rho_{2} \rho_{5}} \mathrm{~S}_{\rho_{1} \rho_{2}} \mathrm{~S}_{\rho_{3} \rho_{4}} \mathrm{~S}_{\rho_{5} \rho_{6}} \\
W_{8}= & \left(\alpha^{\prime}\right)^{4}\left[\frac{\zeta(4)}{4} h^{\rho_{8} \rho_{5}} h^{\rho_{6} \rho_{3}} h^{\rho_{4} \rho_{1}} h^{\rho_{2} \rho_{7}} \mathrm{~S}_{\rho_{1} \rho_{2}} \mathrm{~S}_{\rho_{3} \rho_{4}} \mathrm{~S}_{\rho_{5} \rho_{6}} \mathrm{~S}_{\rho_{7} \rho_{8}}\right. \\
& \left.+\frac{1}{2}\left(\frac{\zeta(2)}{2}\right)^{2}\left(h^{\rho_{4} \rho_{1}} h^{\rho_{2} \rho_{3}} \mathrm{~S}_{\rho_{1} \rho_{2}} \mathrm{~S}_{\rho_{3} \rho_{4}}\right)\left(h^{\rho_{8} \rho_{5}} h^{\rho_{6} \rho_{7}} \mathrm{~S}_{\rho_{5} \rho_{6}} \mathrm{~S}_{\rho_{7} \rho_{8}}\right)\right] \\
W_{10}= & \left(\alpha^{\prime}\right)^{5}\left[\frac{\zeta(5)}{5} h^{\rho_{10} \rho_{7}} h^{\rho_{8} \rho_{5}} h^{\rho_{6} \rho_{3}} h^{\rho_{4} \rho_{1}} h^{\rho_{2} \rho_{9}} \mathrm{~S}_{\rho_{1} \rho_{2}} \mathrm{~S}_{\rho_{3} \rho_{4}} \mathrm{~S}_{\rho_{5} \rho_{6}} \mathrm{~S}_{\rho_{7} \rho_{8}} \mathrm{~S}_{\rho_{9} \rho_{10}}\right. \\
& \left.+\frac{\zeta(2)}{2} \frac{\zeta(3)}{3}\left(h^{\rho_{4} \rho_{1}} h^{\rho_{2} \rho_{3}} \mathrm{~S}_{\rho_{1} \rho_{2}} \mathrm{~S}_{\rho_{3} \rho_{4}}\right)\left(h^{\rho_{10} \rho_{7}} h^{\rho_{8} \rho_{5}} h^{\rho_{4} \rho_{9}} \mathrm{~S}_{\rho_{5} \rho_{6}} \mathrm{~S}_{\rho_{7} \rho_{8}} \mathrm{~S}_{\rho_{9} \rho_{10}}\right)\right],
\end{aligned}
$$

and $\zeta(n)$ is the Riemann $\zeta$-function. Notice that $4 n+2$-form corrections are possible, which is not the case for the corrections involving the bulk Riemann tensor and the second fundamental form [15, 17]. This is not a contradiction, since as we will see in a later section the $4 n+2$-form corrections vanish after dimensional reduction and setting the gauge field in the lower dimension to zero, thus no $4 n+2$-form corrections involving only the transverse scalars appear.

The corrections (3.3), (3.4) can be written even more compactly if we define the trace operation tr over $S_{\rho_{1} \rho_{2}} \cdots S_{\rho_{2 n-1} \rho_{2 n}}$ as in (3.4). In words: start with the index to far right and contract this index with the first index on the $\mathrm{S}$ next to it to the left using $h^{\rho_{2 n} \rho_{2 n-3}}$; then contract the remaining index on $S_{\rho_{2 n-3} \rho_{2 n-2}}$ with the first index on the next $S$ and so on until $S_{\rho_{1} \rho_{2}}$ is reached; finally contract its remaining index $\left(\rho_{2}\right)$ with the remaining index $\left(\rho_{2 n-1}\right)$ on $S_{\rho_{2 n-1} \rho_{2 n}}$ using $h^{\rho_{2} \rho_{2 n-1}}$. This definition is cyclic, which justifies calling it a trace. Using tr the $2 n$-form $2 n$-derivative corrections (3.3), (3.4) can be written

$$
S=T_{9} \int C \exp \left[F+\sum_{n=2}^{5} \frac{\zeta(n)}{n}\left(\alpha^{\prime} \hat{\operatorname{tr} S}\right)^{n}\right] .
$$

The form of this expression is closely related to the $\Gamma$-function, as can be seen by recalling that

$$
-e^{-\gamma z} z \Gamma(-z)=\exp \left(\sum_{n=2}^{\infty} \frac{\zeta(n)}{n} z^{n}\right)=\prod_{l=1}^{\infty}\left(1-\frac{z}{l}\right)^{-1} e^{-\frac{z}{l}},
$$

where $\gamma$ is the Euler constant.

Finally, let us remark that since the only effect of a constant NSNS $B$-field in the string $\sigma$-model is to shift $F \rightarrow F+B$, we can easily modify our expressions to include such a field. To treat the case with a non-constant $B$-field, the fact that string theory is symmetric under the transformation $B \rightarrow B+\partial_{\mu} \Lambda_{\nu}-\partial_{\nu} \Lambda_{\mu}$ should be taken into account. Under this transformation the gauge field on the brane transforms as $A_{\mu} \rightarrow A_{\mu}-\Lambda_{\mu}$. The 
most straightforward way to write invariant expressions is thus to replace $F$ by $F+B$. However, this is not the end of the story, since in addition $H=\mathrm{d} B$ is also invariant under the above symmetry and may appear explicitly. To treat the case with non-constant $B$ fields the results obtained in [31] may be useful. Presumably, there are also corrections which are non-linear in the $\mathrm{RR}$ form fields, $C_{p}$. For non-constant backgrounds there are also corrections involving the bulk Riemann tensor [15, 17]. In addition, corrections involving derivatives of the dilaton $\Phi, B_{\mu \nu}$ and the $C_{p}$ 's are expected.

\section{Corrections to the Born-Infeld term}

In this section we derive the complete expression (at disk level) for the bosonic fourderivative corrections to the Born-Infeld part of the effective action for a D-brane coupled to constant background fields. In particular, the expression involves all orders of the gauge field $F$. The result is obtained via a 3 -loop calculation of the string $\sigma$-model partition function, using the boundary state operator language. The calculations are more involved than the ones performed for the Wess-Zumino term in the previous section. The fermionic modes are now half-integer moded and there are no fermionic zero modes. A complication is the presence of renormalisation scheme dependent terms. Fortunately, we can use the fact that in the course of the determination of the contribution to the Wess-Zumino term one field redefinition was singled out, namely the one that removed the $\left(\psi_{0}\right)^{2}$ (two-form) contribution. Precisely this field redefinition will greatly simplify the corrections to the Born-Infeld part as well. After a tedious calculation, described in more detail in appendix B, one arrives at the following result

$$
\begin{aligned}
S_{\mathrm{BI}}^{(2)}=-T_{9} \int \mathrm{d} \sigma \sqrt{\operatorname{det} h}\left[1+\frac{\left(2 \pi \alpha^{\prime}\right)^{2}}{96}(\right. & -h^{\mu_{4} \mu_{1}} h^{\mu_{2} \mu_{3}} h^{\rho_{4} \rho_{1}} h^{\rho_{2} \rho_{3}} S_{\rho_{1} \rho_{2} \mu_{1} \mu_{2}} S_{\rho_{3} \rho_{4} \mu_{3} \mu_{4}} \\
& \left.\left.+\frac{1}{2} h^{\rho_{4} \rho_{1}} h^{\rho_{2} \rho_{3}} S_{\rho_{1} \rho_{2}} S_{\rho_{3} \rho_{4}}\right)\right]
\end{aligned}
$$

where as before

$$
S_{\rho_{1} \rho_{2} \mu_{1} \mu_{2}}=\partial_{\rho_{1}} \partial_{\rho_{2}} F_{\mu_{1} \mu_{2}}+2 h^{\nu_{1} \nu_{2}} \partial_{\rho_{1}} F_{\left[\mu_{1} \mid \nu_{1}\right.} \partial_{\rho_{2} \mid} F_{\left.\mu_{2}\right] \nu_{2}}
$$

and in addition $S_{\rho_{1} \rho_{2}}:=h^{\mu_{1} \mu_{2}} S_{\rho_{1} \rho_{2} \mu_{1} \mu_{2}}$ (not to be confused with the two-form $\mathrm{S}_{\rho_{1} \rho_{2}}$ which appeared in the previous section). It is straightforward to check that the result (4.1) only contains even powers of $F$, by using $h^{\mu \nu}(-F)=h^{\nu \mu}(F)$ together with $S_{\rho_{1} \rho_{2} \mu_{1} \mu_{2}}(-F)=$ $S_{\rho_{2} \rho_{1} \mu_{2} \mu_{1}}(F)$.

The four-derivative corrections to the Born-Infeld part of the action involving four $F$ 's and four derivatives have been known for a long time. These corrections can be extracted from the four-string scattering amplitude (see e.g. [29]) and have the form [22]

$$
\begin{aligned}
& S_{(\partial F)^{4}}=-T_{9} \frac{\alpha^{\prime 2} \pi^{2}}{24} \int\left[\frac{1}{8} \operatorname{tr}\left(\partial_{\rho} F \partial^{\rho} F\right) \operatorname{tr}\left(\partial_{\lambda} F \partial^{\lambda} F\right)+\frac{1}{4} \operatorname{tr}\left(\partial_{\rho} F \partial_{\lambda} F\right) \operatorname{tr}\left(\partial^{\rho} F \partial^{\lambda} F\right)\right. \\
& \left.-\frac{1}{2} \operatorname{tr}\left(\partial_{\lambda} F \partial_{\rho} F \partial^{\lambda} F \partial^{\rho} F\right)-\operatorname{tr}\left(\partial_{\lambda} F \partial_{\rho} \partial^{\rho} F \partial^{\lambda} F\right)\right] .
\end{aligned}
$$


The integrand can be rewritten in terms of the well known eight-index $t_{8}$-tensor as $-\frac{1}{16} t_{8} \partial_{\rho} F \partial_{\lambda} F \partial^{\lambda} F \partial^{\rho} F$ (the definition of $t_{8}$ is given in appendix B). To check whether our result (4.1) correctly reduces to (4.3) when restricting to the $\mathcal{O}\left(F^{4}\right)$ terms is rather involved, since the two expressions need only be equal up to terms which can be removed by field redefinitions. (This ambiguity also accounts for the seeming discrepancy between (4.3) and the expression in [22].) It turns out that the four different types of terms entering in (4.3) form a basis for any term constructed out of four derivatives and four F's modulo field redefinitions, integrations by parts and use of the Bianchi identity. This fact is shown in appendix $\mathrm{B}$. Armed with this result it is fairly straightforward to show that the two expressions do indeed agree. This is a non-trivial check of our result. For more details see appendix B. Another test of our result is to check that it transforms correctly under the Seiberg-Witten map [11. We hope to return to this question elsewhere [32].

As before, the dependence on constant background fields can be taken care of by replacing $F$ by $F+B$ and (in the string frame) introduce an overall factor of $e^{-\Phi}$. So far we have only discussed derivative corrections at disk level in the string-coupling perturbation expansion. In addition there should also be contributions from loops and non-perturbative effects. Such corrections were discussed in [33] and were argued to appear for the $(\partial F)^{4}$ part of the action for the D3-brane as an overall multiplicative function. This is presumably also true for our four-derivative result involving all orders of $F$.

\section{Lower-dimensional cases}

The results in sections 3 and 1 are valid for the nine-brane case. To obtain the corrections to the lower-dimensional branes we need to dimensionally reduce the above action (Tduality). We will now change notation slightly and label ten-dimensional indices by $M, N, \ldots$, world-volume indices by $\mu, \nu, \ldots$, and indices in the directions normal to the brane by $i, j, \ldots$. The embedding of the brane into the ten-dimensional target space is described by the elements $\partial_{\mu} Y^{M}$, which span a local frame of the tangent bundle and the objects $\xi_{M}^{i}$ which play a similar role for the normal bundle (for more details, see e.g. [17]). World-volume indices are raised and lowered with the induced metric $g_{\mu \nu}:=\delta_{M N} \partial_{\mu} Y^{N} \partial_{\nu} Y^{M}$, indices in the directions normal to the brane with $\delta_{i j}$ and tendimensional indices with $\delta_{M N}$.

The fundamental quantity describing the embedding is the second fundamental form, $\Omega_{\mu \nu}^{M}$, defined as the covariant derivative of $\partial_{\mu} Y^{M}$; for a flat background we thus have

$$
\Omega_{\mu \nu}^{M}=\partial_{\mu} \partial_{\nu} Y^{M}-\Gamma_{\mathrm{T}_{\mu \nu}^{\lambda}}^{\lambda} \partial_{\lambda} Y^{M}
$$

where $\Gamma_{\mathrm{T}}^{\lambda}{ }_{\mu \nu}$ is the connection constructed from the induced metric. More explicitly, we find $\Omega_{\mu \nu}^{M}=\left(\delta_{L}^{M}-\partial_{\lambda} Y^{M} g^{\lambda \rho} \partial_{\rho} Y^{K} \delta_{K L}\right) \partial_{\mu} \partial_{\nu} Y^{L}=: P_{L}^{M} \partial_{\mu} \partial_{\nu} Y^{L}$. It can easily be shown that $P_{L}^{M}$ is a projection matrix, $P_{N}^{M} P_{L}^{N}=P_{L}^{M}$. Since $\delta^{M N}=\partial_{\lambda} Y^{M} g^{\lambda \rho} \partial_{\rho} Y^{N}+\xi_{i}^{M} \delta^{i j} \xi_{j}^{N}$, we find that $\xi_{i}^{M} \delta^{i j} \xi_{j}^{N}=P^{M N}$. Is is easy to check that the projection of $\Omega_{\mu \nu}^{M}$ onto the tangent bundle, $\partial_{\rho} Y^{N} \delta_{N M} \Omega_{\mu \nu}^{M}$, vanishes. We introduce the notation $\hat{\Omega}_{\mu \nu}^{i}:=\xi_{M}^{i} \Omega_{\mu \nu}^{M}$ for the second fundamental form projected onto the normal bundle. 
The $\alpha^{\prime 2}$ corrections to the Wess-Zumino term involving the Riemann tensor are [15, 17

$$
S_{R^{2}}=T_{p} \int C \wedge e^{F} \wedge \frac{\left(2 \pi \alpha^{\prime}\right)^{2}}{96}\left[\operatorname{tr}\left(R_{\mathrm{T}} \wedge R_{\mathrm{T}}\right)-\operatorname{tr}\left(R_{\mathrm{N}} \wedge R_{\mathrm{N}}\right)\right]
$$

where the subscripts $\mathrm{T}$ and $\mathrm{N}$ refer to the normal and tangent bundles, respectively. In a flat background we have the relations

$$
\begin{aligned}
\left(R_{\mathrm{T}}\right)_{\mu \nu \rho \lambda} & =\delta_{i j} \hat{\Omega}_{\mu \rho}^{i} \hat{\Omega}_{\nu \lambda}^{j}-(\mu \leftrightarrow \nu), \\
\left(R_{\mathrm{N}}\right)_{\mu \nu}{ }^{i j} & =g^{\rho \lambda} \hat{\Omega}_{\mu \rho}^{i} \hat{\Omega}_{\nu \lambda}^{j}-(\mu \leftrightarrow \nu) .
\end{aligned}
$$

The above corrections are thus expressed in terms of the second fundamental form. In the static gauge, $Y^{\mu}=x^{\mu}$ and $Y^{i}=X^{i}(x)$, we find

$$
S_{R}^{(2)}=T_{p} \frac{\left(2 \pi \alpha^{\prime}\right)^{2}}{48} \int C e^{F} P^{i_{1} i_{2}} P^{i_{3} i_{4}} g^{\rho_{1} \rho_{4}} g^{\rho_{2} \rho_{3}} \partial_{\rho_{1}} \mathrm{~d} X_{i_{1}} \partial_{\rho_{2}} \mathrm{~d} X_{i_{2}} \partial_{\rho_{3}} \mathrm{~d} X_{i_{3}} \partial_{\rho_{4}} \mathrm{~d} X_{i_{4}} .
$$

Next we will investigate whether these corrections are correctly reproduced by the dimensional reduction of our result, (3.2). When reducing, the gauge field $F_{M N}$ splits into $F_{\mu \nu}$ and $F_{\mu i}=\partial_{\mu} X_{i}\left(F_{i j} \equiv 0\right)$. Thus,

$$
h_{M N}=\left(\begin{array}{cc}
(1+F)_{\mu \nu} & \partial_{\mu} X_{j} \\
-\partial_{\nu} X_{i} & \delta_{i j}
\end{array}\right)
$$

The inverse, $h^{M N}$, becomes

$$
h^{M N}=\left(\begin{array}{cc}
\tilde{h}^{\mu \nu} & -\tilde{h}^{\mu \lambda} \partial_{\lambda} X^{j} \\
\partial_{\lambda} X^{i} \tilde{h}^{\lambda \nu} & \delta^{i j}-\partial_{\lambda} X^{i} \tilde{h}^{\lambda \rho} \partial_{\rho} X^{j}
\end{array}\right) .
$$

Here $\tilde{h}^{\mu \nu}=\left(\frac{1}{\eta+F+\partial X^{i} \partial X_{i}}\right)^{\mu \nu}$. When $F_{\mu \nu}=0, \tilde{h}^{\mu \nu}$ reduces to the inverse of the induced metric, $g^{\mu \nu}$. If we concentrate on the part of $\mathrm{S} \wedge \mathrm{S}$ in (3.2) which has four indices in the world-volume directions, we see that only the $(\partial F)^{4}$ part contributes when $F_{\mu \nu}=0$. Furthermore, observing that (when $F$ is zero) $h^{i j}=P^{i j}$ we find complete agreement with (5.4).

When reducing the zeroth order Wess-Zumino term from ten to nine dimensions the part of $e^{F}$ with one index transverse to the world volume gets reinterpreted as part of the pullback of $C$ to nine dimensions by T-duality [7]. We have not investigated how this statement generalises for the corrections considered in section 3 .

Let us now look at the higher-degree corrections. When $F_{\mu \nu}=0$, one can easily show that $S_{\rho_{1} \rho_{2} \mu_{1} \mu_{2}}=-S_{\rho_{2} \rho_{1} \mu_{1} \mu_{2}}$, which implies that the six- and ten-form corrections in (3.4) vanish when we put the gauge field to zero. The eight-form corrections are, however, non-vanishing. These should be compared with the eight-form corrections involving the second fundamental form. Starting from the expression [15]

$$
S_{R}=T_{p} \int C \wedge e^{F} \wedge \frac{\sqrt{\hat{\mathcal{A}}\left(4 \pi^{2} \alpha^{\prime} R_{\mathrm{T}}\right)}}{\sqrt{\hat{\mathcal{A}}\left(4 \pi^{2} \alpha^{\prime} R_{\mathrm{N}}\right)}},
$$


where

$$
\sqrt{\hat{\mathcal{A}}\left(4 \pi^{2} \alpha^{\prime} R\right)}=1-\frac{\pi^{2} \alpha^{\prime 2}}{24} \operatorname{tr} R^{2}-\frac{\pi^{4} \alpha^{\prime 4}}{720} \operatorname{tr}\left(R^{4}\right)+\frac{\pi^{4} \alpha^{\prime 4}}{192}\left(\operatorname{tr}\left(R^{2}\right)\right)^{2}+\ldots,
$$

and using the results (5.3) it is straightforward to check that also the eight-form corrections are correctly reproduced. In [17 $\alpha^{2}$ corrections to the Born-Infeld part of the action involving the second fundamental form were discussed. It should also be possible to check if these corrections are correctly reproduced by our result.

\section{Connection to non-symmetric gravity}

In the corrections obtained in sections 3 and 4 only the combination $h_{\mu \nu}=(1+F)_{\mu \nu}$ appears (derivatives on $F$ can be replaced by derivatives on $h$ ). In addition, the form of $S_{\rho_{1} \rho_{2} \mu_{1} \mu_{2}}$ is reminiscent of the usual expression for the Riemann tensor. Let us see if it is possible to make this analogy more quantitative. The Riemann tensor, $T_{\rho \mu \nu}^{\lambda}$, for a non-symmetric metric is given by (see e.g. [34; the ordering of indices is important)

$$
T_{\rho \mu \nu}^{\lambda}=\partial_{\mu} \Upsilon_{\rho \nu}^{\lambda}-\Upsilon_{\rho \mu}^{\sigma} \Upsilon_{\sigma \nu}^{\lambda}-(\mu \leftrightarrow \nu)
$$

where $\Upsilon_{\rho \nu}^{\lambda}$ is the connection. Let us further define $\Upsilon_{\lambda \rho \nu}:=h_{\lambda \sigma} \Upsilon^{\sigma}{ }_{\rho \nu}$ and $T_{\lambda \rho \mu \nu}:=$ $h_{\lambda \sigma} T_{\rho \mu \nu}^{\sigma}$. If we choose $\Upsilon_{\lambda \rho \nu}=\partial_{\rho} h_{\lambda \nu}=\partial_{\rho} F_{\lambda \nu}$, then a short calculation leads to $T_{\lambda \rho \mu \nu}=$ $S_{\lambda \rho \mu \nu}$. Thus, $S_{\lambda \rho \mu \nu}$ can be interpreted as the Riemann tensor for a non-symmetric metric.

The following useful relations for the derivatives of $h_{\rho_{1} \rho_{2}}$ and $S_{\rho_{1} \rho_{2} \mu_{1} \mu_{2}}$ hold:

$$
\begin{aligned}
& \partial_{\mu} h_{\rho_{1} \rho_{2}}-h_{\rho_{1} \lambda} \Upsilon_{\rho_{2} \mu}^{\lambda}-\Upsilon_{\mu \rho_{1} \lambda} \delta_{\rho_{2}}^{\lambda}=0 \\
& \partial_{\left[\mu_{3}\right.} S_{\left.\left|\rho_{1} \rho_{2}\right| \mu_{1} \mu_{2}\right]}-S_{\rho_{1} \lambda\left[\mu_{1} \mu_{2}\right.} \Upsilon^{\lambda}{ }_{\left.\left|\rho_{2}\right| \mu_{3}\right]}-\Upsilon_{\left[\mu_{3} \mid \rho_{1} \lambda\right.} S_{\left.\rho_{2} \mid \mu_{1} \mu_{2}\right]}^{\lambda}=0 \text {. }
\end{aligned}
$$

The first equation is analogous to the one showing that the metric is covariantly constant for the case of a symmetric metric, whereas the second one is similar to the Bianchi identity for the usual Riemann tensor. Using the second equation in (6.2), it is straightforward to check that $\mathrm{d}\left[\hat{\operatorname{tr}}\left(\mathrm{S}^{k}\right)\right]=0$, thus showing that the corrections (3.4) are all closed.

Some further interesting relations are obtained upon dimensional reduction. We have seen in the previous section that when the gauge field in the lower dimension is zero we obtain the inverse of the induced metric from the part of $h^{M N}$ with both indices longitudinal to the world volume. Similarly, from the ${ }^{\rho}{ }_{\mu \nu}$ part of $\Upsilon^{R}{ }_{M N}$ we obtain $\Gamma_{\mathrm{T}}^{\lambda}{ }_{\mu \nu}$, and from the ${ }_{\mu \nu}^{i}$ part we get $-\Omega_{\mu \nu}^{i}$ (the sign can be removed by redefining $X^{i} \rightarrow-X^{i}$ ). To obtain these relations we used the static gauge.

So far we have discussed a second order formalism, i.e. one where the connection is expressed in terms of the metric. There should also exist a first order formalism in which the Riemann tensor in the action is taken to depend on the connection, and the relation between the connection and the metric follows from the equation of motion for the connection. In the usual case one can always go between the two formulations by varying the Einstein-Hilbert action in the form $\int \sqrt{-g} g^{\mu \nu} R_{\mu \nu}(\Gamma)$. In our case it is not a priori clear which action to use as a replacement for the this action. Notice for instance that one 
can construct more than one independent "Ricci" tensor, e.g. $T_{\rho_{1} \rho_{2}}:=h^{\mu_{1} \mu_{2}} T_{\rho_{1} \rho_{2} \mu_{1} \mu_{2}}$ and $\tilde{T}_{\rho_{1} \mu_{1}}=h^{\rho_{2} \mu_{2}} T_{\rho_{1} \rho_{2} \mu_{1} \mu_{2}}$ (see [34] for more details). Since the $\mathcal{O}\left(\alpha^{\prime}\right)$ corrections vanish for the superstring case we get no clues from the string theory calculation. If the relation to non-symmetric gravity is valid beyond supersymmetry then a calculation in the bosonic string may lead to some insight. Unfortunately this calculation has not been done to all orders in $F$.

We would like to stress that although the above results are very suggestive it may be that the structure is only present for the terms we have considered; it would therefore be interesting to determine some other corrections, to see whether the structure persists. We would also like to point out that the problems associated with theories with a nonsymmetric metric raised in [34] are not directly applicable to our situation. In [34] only metrics of the form $\delta+B$ were discussed, which leads to problems with the $\Lambda$-symmetry, $B \rightarrow B+\mathrm{d} \Lambda$. Since we also have $F$ at our disposal, these problems are circumvented. Also, we discuss four-derivative corrections, whereas the focus in [34 was on two-derivative terms. Theories with a non-symmetric metric have also been dicussed within the context of D-brane effective actions in [35].

Is it possible to take the above discussion one step further and somehow also include a curved background metric and in this way incorporate the corrections involving the Riemann curvature determined in [15]? For the nine-brane case one might try expressing everything in terms of $h_{\mu \nu}=G_{\mu \nu}+F_{\mu \nu}$, where $G$ is the background metric, and construct a $\Upsilon_{\lambda \rho \nu}$ which is such that it reduces to $\Gamma_{\lambda \rho \nu}$ (possibly up to a multiplicative constant) when $h_{\mu \nu}=G_{\mu \nu}$, so that $T_{\lambda \rho \mu \nu}$ reduces to $R_{\lambda \rho \mu \nu}$. The answer appears to be no. It is possible to find a $\Upsilon_{\lambda \rho \nu}$ which does the trick for the four-derivative corrections, but the same expression will give erroneous results for the higher-form corrections to the WessZumino term.

\section{Acknowledgements}

The author would like to thank Michael Green and Kasper Peeters for discussions. This work was supported by the European Commission under the contract FMBICT983302. The author has also benefitted from the PPARC grant PPA/G/S/1998/00613.

\section{Appendices}

\section{A Conventions}

For convenience we have collected our conventions in this appendix. The boundary of the disk is parameterised by the variable $\sigma$ which takes values from 0 to $2 \pi$. The string coordinate on the boundary is $X^{\mu}(\sigma)$ and has the following expansion in terms of the $\alpha_{n}^{\mu}$ 
and $\tilde{\alpha}_{n}^{\mu}$ oscillators

$$
X^{\mu}(\sigma)=x^{\mu}+i \sqrt{\frac{\alpha^{\prime}}{2}} \sum_{n \neq 0}\left(\frac{\alpha_{n}^{\mu}}{n} e^{i n \sigma}+\frac{\tilde{\alpha}_{n}^{\mu}}{n} e^{-i n \sigma}\right) .
$$

The commutation relations are as usual $\left[\alpha_{m}^{\mu}, \alpha_{n}^{\nu}\right]=m \eta^{\mu \nu} \delta_{m+n}$ and $\left[\tilde{\alpha}_{m}^{\mu}, \tilde{\alpha}_{n}^{\nu}\right]=m \eta^{\mu \nu} \delta_{m+n}$, from which it follows that $\left[X^{\mu}(\sigma), X^{\nu}(\xi)\right]=0$. The boundary state $|B\rangle$ 23, 24, satisfies (for the nine-brane case) $P_{\mu}|B\rangle=0$ (where $P_{\mu}$ is the string momentum). The explicit expression for $|B\rangle$ is not needed in this paper; it can be found e.g. in [26]. In the presence of a gauge field $A_{\mu}(X)$, with field strength $F_{\mu \nu}(X)=\frac{\partial}{\partial X^{\mu}} A_{\nu}(X)-\frac{\partial}{\partial X^{\nu}} A_{\mu}(X)$, the above condition is replaced by $\left[2 \pi \alpha^{\prime} P_{\mu}+F_{\mu \nu}(X) \partial_{\sigma} X^{\nu}\right]|B(F(X))\rangle=0$. Throughout the paper we absorb a factor of $2 \pi \alpha^{\prime}$ in $F_{\mu \nu}$; what we call $F_{\mu \nu}$ is thus really $2 \pi \alpha^{\prime} F_{\mu \nu}$. When we refer to derivative corrections we mean corrections with additional factors of $\alpha^{\prime}$.

We use an unconventional normalisation for the fermionic coordinates, $\Psi_{A}^{\mu}, A=$ ,-+ . The canonical anti-commutation relations between the fermions are taken to be $\left\{\Psi_{A}^{\mu}(\sigma), \Psi_{B}^{\nu}(\xi)\right\}=-i \pi \alpha^{\prime} \delta^{\mu \nu} \delta_{A B} \delta(\sigma-\xi)$. The mode expansions are

$$
\begin{aligned}
& \mathrm{R}:\left\{\begin{array}{l}
\Psi_{-}^{\mu}(\sigma)=\sqrt{-\frac{i \alpha^{\prime}}{2}} \sum_{n \in \mathbb{Z}} d_{n}^{\mu} e^{i n \sigma}, \\
\Psi_{+}^{\mu}(\sigma)=\sqrt{-\frac{i \alpha^{\prime}}{2}} \sum_{n \in \mathbb{Z}} \tilde{d}_{n}^{\mu} e^{-i n \sigma},
\end{array}\right. \\
& \mathrm{NS}:\left\{\begin{array}{l}
\Psi_{-}^{\mu}(\sigma)=\sqrt{-\frac{i \alpha^{\prime}}{2}} \sum_{r \in \mathbb{Z}+\frac{1}{2}} b_{r}^{\mu} e^{i r \sigma}, \\
\Psi_{+}^{\mu}(\sigma)=\sqrt{-\frac{i \alpha^{\prime}}{2}} \sum_{r \in \mathbb{Z}+\frac{1}{2}} \tilde{b}_{r}^{\mu} e^{-i r \sigma},
\end{array}\right.
\end{aligned}
$$

with $\left\{d_{m}^{\mu}, d_{n}^{\nu}\right\}=\delta^{\mu \nu} \delta_{m+n}$ and $\left\{b_{r}^{\mu}, b_{s}^{\nu}\right\}=\delta^{\mu \nu} \delta_{r+s}$ in the NS and R sectors, respectively. We also use the notation $\Psi^{\mu}=\Psi_{-}^{\mu}+i \eta \Psi_{+}^{\mu}$ and $\bar{\Psi}^{\mu}=\Psi_{-}^{\mu}-i \eta \Psi_{+}^{\mu}$. The boundary state $|B\rangle$ satisfies $\bar{\Psi}^{\mu}|B\rangle=0$. In the presence of a gauge field $A_{\mu}(X)$, the boundary condition reads $\left[\bar{\Psi}_{\mu}-F_{\mu \nu}(X) \Psi^{\nu}\right]|B(F(X))\rangle=0$. To implement the GSO projection one has to take certain linear combinations of the two spin-structure sectors. The details are not important to us since the derivative corrections are not sensitive to such considerations.

We work in euclidian signature (our results can, however, easily be transformed to Minkowski signature). Our normalisation of the boundary term in the $\sigma$-model is

$$
S=\frac{i}{2 \pi \alpha^{\prime}} \int d \sigma\left(\partial_{\sigma} X^{\mu} A_{\mu}(X)-\frac{1}{2} \Psi^{\mu} \Psi^{\nu} F_{\mu \nu}(X)\right) .
$$

When convenient, we use superfields; our conventions are: $D=\theta \partial_{\sigma}-\partial_{\theta}, \phi^{\mu}=X^{\mu}+\theta \Psi^{\mu}$. In the superfield language the boundary term can be written $\frac{i}{2 \pi \alpha^{\prime}} \int d \sigma \mathrm{d} \theta D_{\sigma} \phi^{\mu} A_{\mu}(\phi)$. and

Our conventions for differential forms are as follows: $X_{p}=\frac{1}{p !} X_{\mu_{1} \cdots \mu_{p}} \mathrm{~d} x^{\mu_{1}} \wedge \cdots \wedge \mathrm{d} x^{\mu_{p}}$

$$
X_{p} \wedge Y_{q}=\frac{1}{p ! q !} X_{\left[\mu_{1} \cdots \mu_{p}\right.} Y_{\left.\mu_{p+1} \cdots \mu_{p+q}\right]} \mathrm{d} x^{\mu_{1}} \wedge \cdots \wedge \mathrm{d} x^{\mu_{p+q}}
$$

We often suppress the wedge product. In the cases of branes with lower than maximal dimension we use the following index conventions. Indices longitudinal to the brane (i.e. in the world-volume directions) are denoted $\mu, \nu, \ldots$, indices in the normal directions $i, j, \ldots$ and finally ten-dimensional bulk indices are labelled by $M, N, \ldots$. For the nine-brane we use $\mu, \nu, \ldots$ to denote both bulk and brane indices, since D9-branes are space filling. 


\section{B Technical details}

In this appendix we will give some technical details of the calculations underlying our results. Because of the complexity of the problem we will focus on the main steps.

\section{B.1 Corrections to the Wess-Zumino term}

In this subsection we will discuss the method we used to derive the expressions for the corrections to the Wess-Zumino term (3.3), (3.4) and the field redefinitions needed to remove the two-form part at order $\alpha^{\prime 2}$. Here we will only consider the terms which have non-logarithmic finite parts. This means that we exclude terms where two $\phi$ 's within the same factor are contracted, since $\left[G_{12}^{\mu \nu}\right]_{1 \rightarrow 2}$ contains a multiplicative $\ln \epsilon$ factor. In almost all expressions below we suppress an overall contraction of $\langle C|\cdots| B(F)\rangle$.

The first step of the calculations is to expand the exponential in (2.3) using (2.13), insert the result into (2.6) and collect terms of the same order in derivatives. Next one uses the method outlined in section 2, to rewrite the resulting expressions in terms of propagators by calculating the various correlation functions. In this phase it is imperative to use the fact that $F_{\mu \nu}$ satisfies the Bianchi identity

$$
\partial_{[\rho} F_{\mu \nu]}=0 \Leftrightarrow \partial_{\rho} F_{\mu \nu}+\partial_{\mu} F_{\nu \rho}+\partial_{\nu} F_{\rho \mu}=0
$$

Various symmetry properties of the propagator are also useful, such as $G_{12}^{\mu \nu}=G_{21}^{\nu \mu}$ and $D_{1} G_{12}^{\mu \nu}=\left(\theta_{1}-\theta_{2}\right) \partial_{1} D_{12}^{\mu \nu}$. The latter result holds only in the $\mathrm{R}$ sector and follows from the fact that in this sector $K_{12}^{\mu \nu}=\partial_{2} D_{12}^{\mu \nu}=-\partial_{1} D_{12}^{\mu \nu}$. Relabelling of indices and coordinates, as well as integration by parts can also profitably be used to relate various terms. Also, for the $\int \mathrm{d} \sigma \partial_{\sigma} \phi \phi^{2} \partial F$ factors, the part with two fermionic zero modes vanishes since this term is a total derivative.

Let us now discuss the $\alpha^{\prime 2}$ corrections. It is straightforward to check that the $\partial^{3} F \partial F$ and $\partial^{4} F$ contributions only involve logarithmic terms. Furthermore, the terms with no fermionic zero modes vanish by the general result given in section 2. Let us therefore start by discussing the terms with two zero modes. For this case the $\partial^{2} F \partial^{2} F$ terms do not have any non-logarithmic finite parts. Examples of terms with non-logarithmic finite parts are:

$$
\begin{aligned}
& \underbrace{\partial F}_{\text {는 }} \partial^{2} F=\frac{1}{4}\left(\frac{-i}{2 \pi \alpha^{\prime}}\right)^{3} \psi_{0}^{\eta} \psi_{0}^{\delta} \int \mathrm{d} \sigma \partial_{1} D_{13}^{\mu_{1} \lambda_{1}} D_{23}^{\mu_{2} \lambda_{2}} \partial_{2} D_{12}^{\nu_{1} \nu_{2}} D_{12}^{\rho_{1} \rho_{2}} \partial_{\rho_{1}} F_{\mu_{1} \nu_{1}} \partial_{\rho_{2}} F_{\mu_{2} \nu_{2}} \partial_{\lambda_{1}} \partial_{\lambda_{2}} F_{\eta \delta} \text {, }
\end{aligned}
$$

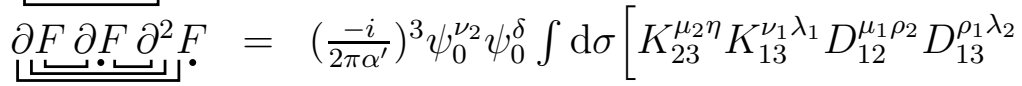

$$
\begin{aligned}
& \left.+K_{12}^{\mu_{1} \mu_{2}} K_{13}^{\nu_{1} \eta} D_{13}^{\rho_{1} \lambda_{1}} D_{23}^{\rho_{2} \lambda_{2}}\right] \partial_{\rho_{1}} F_{\mu_{1} \nu_{1}} \partial_{\rho_{2}} F_{\mu_{2} \nu_{2}} \partial_{\lambda_{1}} \partial_{\lambda_{2}} F_{\eta \delta} .
\end{aligned}
$$

In addition, there are also two different types of contractions of the $\partial F \partial F \partial F \partial F$ terms with non-logarithmic finite parts. A few words about our notation are in order. The dots in the above expressions indicate zero modes. Furthermore, $\mathrm{d} \sigma$ is short-hand notation for $\mathrm{d} \sigma_{1} \cdots \mathrm{d} \sigma_{k}$, where $k$ equals the number of $F$ 's. All integrations range from 0 to $2 \pi$. The contractions and the placement of dots are schematical. For instance, a line that seems 
to end on an $F$ is not necessarily a contraction with an index on that $F$, it could also be a contraction with an index on a partial derivative acting on that $F$.

To perform the integration over the $\sigma^{\prime}$ 's, one uses $\int \mathrm{d} \sigma e^{i n \sigma}=2 \pi \delta_{n, 0}$. As an example, for the second expression in $(\mathbb{B . 2})$ one gets

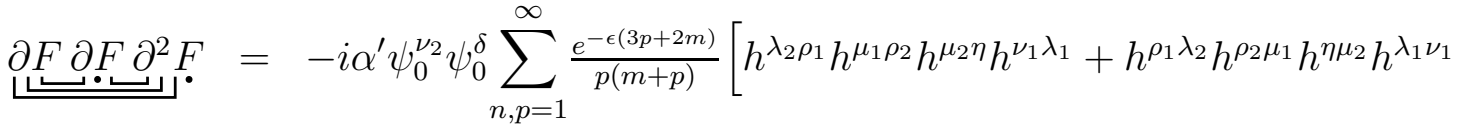

$$
\begin{aligned}
& \left.-h^{\eta \nu_{1}} h^{\mu_{2} \mu_{1}} h^{\lambda_{2} \rho_{2}} h^{\rho_{1} \lambda_{1}}-h^{\nu_{1} \eta} h^{\mu_{1} \mu_{2}} h^{\rho_{2} \lambda_{2}} h^{\lambda_{1} \rho_{1}}\right] \\
& \times \partial_{\rho_{1}} F_{\mu_{1} \nu_{1}} \partial_{\rho_{2}} F_{\mu_{2} \nu_{2}} \partial_{\lambda_{1}} \partial_{\lambda_{2}} F_{\eta \delta} \text {. }
\end{aligned}
$$

The sums arising from the integrations are evaluated in appendix Q. Since it is known that $\langle C \mid B(F)\rangle \rightarrow T_{9} C e^{F}$, and that only the zero mode part of $|B(F)\rangle$ contributes to this result, one can read off the rule $\left\langle C\left|\left(\psi_{0}^{\mu} \psi_{0}^{\nu} X_{\mu \nu}\right)^{k}\right| B(F)\right\rangle \rightarrow T_{9}\left(-2 i \alpha^{\prime}\right)^{k} C e^{F} X^{k}$. This rule implies that the explicit form of $|C\rangle$ is not needed in this paper and also gives the relation between the number of zero modes and the form degree of the resulting correction. It furthermore shows that the above two-form expressions, which are all of the form $\psi_{0}^{\mu} \psi_{0}^{\nu} X_{\mu \nu}$, can be removed by the field redefinition $\delta F=2 i \alpha^{\prime} X$, which is an expression of order $\alpha^{2}$. Whether this can in turn be interpreted as a redefinition of $A_{\mu}$ depends on the renormalisation scheme but there has to exist a scheme in which it is possible; see section 2 for further discussion of this point.

The $\alpha^{\prime 2}$ four-form four-derivative corrections are easier to calculate than the two-form four-derivative ones, since there are more zero-modes and consequently fewer propagators. In fact, without too much additional effort the corresponding $2 n$-form $2 n$-derivative corrections can also be determined. The divergent parts of these expressions are removed by the terms induced by the first order field redefinition (2.15). We will not give the details here. Instead, we will perform one sample calculation of the finite part for one particular 6-form 6-derivative correction, namely the $(\partial F)^{4} \partial^{2} F$ term. The calculation proceeds as follows:

$$
\begin{aligned}
& \partial \underset{\bullet}{F} \partial \underset{\cdot F}{F} \underset{\bullet}{F} \underset{\bullet}{F} \partial^{2} \underset{*}{F}=\frac{5}{5 !}\left(\frac{-i}{2 \pi \alpha^{\prime}}\right)^{5} \frac{1}{2^{2}} \\
& \times \int \mathrm{d} \sigma\left\langle C\left|\left(\psi_{0}^{\nu_{1}} \psi_{0}^{\nu_{2}} \psi_{0}^{\nu_{3}} \psi_{0}^{\nu_{4}} \psi_{0}^{\eta} \psi_{0}^{\delta}\right) \Psi^{\mu_{1}} X^{\rho_{1}} \Psi^{\mu_{2}} X^{\rho_{2}} \Psi^{\mu_{3}} X^{\rho_{3}} \Psi^{\mu_{4}} X^{\rho_{4}} X^{\lambda_{1}} X^{\lambda_{2}}\right| B(F)\right\rangle \\
& \times \partial_{\rho_{1}} F_{\mu_{1} \nu_{1}} \partial_{\rho_{2}} F_{\mu_{2} \nu_{2}} \partial_{\rho_{3}} F_{\mu_{3} \nu_{3}} \partial_{\rho_{4}} F_{\mu_{4} \nu_{4}} \partial_{\lambda_{1} \lambda_{2}} F_{\eta \delta} \\
& =\frac{1}{2^{2}}\left(\frac{-i}{2 \pi \alpha^{\prime}}\right)^{5}\left(\psi_{0}\right)^{6} \int \mathrm{d} \sigma\left[K_{12}^{\mu_{1} \mu_{2}} K_{34}^{\mu_{3} \mu_{4}} D_{15}^{\rho_{1} \lambda_{1}} D_{35}^{\rho_{3} \lambda_{2}} D_{24}^{\rho_{2} \rho_{4}}\right](\partial F)^{4} \partial^{2} F \\
& =\frac{1}{2^{2}}\left(\frac{-i}{2 \pi \alpha^{\prime}}\right)^{5}\left(2 \pi \alpha^{\prime}\right)^{5}\left(\psi_{0}\right)^{6}\left[h^{\mu_{1} \mu_{2}} h^{\mu_{4} \mu_{3}} h^{\lambda_{1} \rho_{1}} h^{\rho_{3} \lambda_{2}} h^{\rho_{2} \rho_{4}}\right. \\
& \left.+h^{\mu_{2} \mu_{1}} h^{\mu_{3} \mu_{4}} h^{\rho_{1} \lambda_{1}} h^{\lambda_{2} \rho_{3}} h^{\rho_{4} \rho_{2}}\right] \sum_{n=1} \frac{e^{-5 \epsilon n}}{n^{3}}(\partial F)^{4} \partial^{2} F .
\end{aligned}
$$

We would like to stress that we only kept the terms with non-logarithmic finite parts, which is why we discarded some of the possible contractions. We have also suppressed the $\langle C|\cdots| B(F)\rangle$ contraction in the last two expressions. By relabelling of indices one can easily show that in the last expression in (B.4) the two $h^{5}$ terms are equal. Finally, 
removing the regulator and using the rule $\left\langle C\left|\left(\psi_{0}^{\mu} \psi_{0}^{\nu} X_{\mu \nu}\right)^{k}\right| B(F)\right\rangle \rightarrow T_{9}\left(-2 i \alpha^{\prime}\right)^{k} C e^{F} X^{k}$, reproduces the $(\partial F)^{4} \partial^{2} F$ part of $W_{6}$ given in (3.4).

\section{B.2 Corrections to the Born-Infeld term}

In this subsection we will give some details of the calculation of the $\alpha^{2}$ four-derivative corrections to the Born-Infeld action. The first step is to expand the exponential in (2.3), (2.4) using (2.13) and collect terms of the same order in derivatives and rewrite the expressions in terms of propagators by calculating the various correlation functions (or, equivalently, by writing down the various Feynman diagrams). The expressions greatly simplify as a consequence of the Bianchi identity, $\partial_{[\rho} F_{\mu \nu]}=0$. At this stage of the calculation various properties of the propagator are also useful, such as

$$
\begin{aligned}
& G_{12}^{\mu \nu}=G_{21}^{\nu \mu}, \\
& \int \mathrm{d} \sigma_{1} G_{12}^{\mu \nu}=0, \\
& D_{1} G_{12}^{\mu \nu}=-D_{2} G_{12}^{\mu \nu}, \\
& D_{1} G_{12}^{\mu \nu} D_{2} G_{12}^{\eta \delta}=D_{1} G_{12}^{\eta \delta} D_{2} G_{12}^{\mu \nu} .
\end{aligned}
$$

The second condition above implies that (in a diagrammatical approach) only diagrams (not neccessarily connected) whose parts are one-particle irreducible contribute. Relabelling of indices and integrations by parts also leads to a reduction of the number of independent terms.

The $\alpha^{2}$ corrections can be classified according to how the index contractions between the different $\partial^{n} F$ 's are performed. The contributions with non-logarithmic finite parts become (after simplifications)

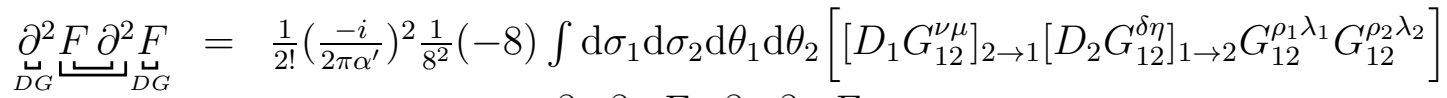

$$
\begin{aligned}
& \times \partial_{\rho_{1}} \partial_{\rho_{2}} F_{\mu \nu} \partial_{\lambda_{1}} \partial_{\lambda_{2}} F_{\eta \delta}
\end{aligned}
$$

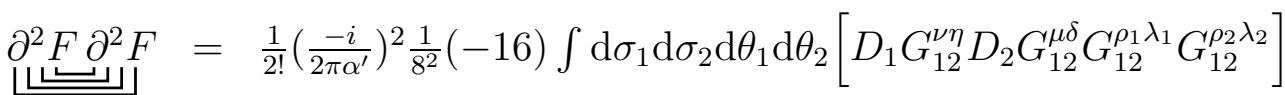

$$
\begin{aligned}
& \times \partial_{\rho_{1}} \partial_{\rho_{2}} F_{\mu \nu} \partial_{\lambda_{1}} \partial_{\lambda_{2}} F_{\eta \delta}
\end{aligned}
$$

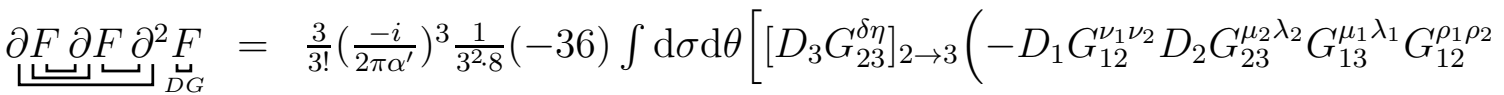

$$
\begin{aligned}
& \left.\left.+\frac{1}{2} D_{1} G_{12}^{\nu_{1} \mu_{2}} D_{2} G_{12}^{\mu_{1} \nu_{2}} G_{13}^{\rho_{1} \lambda_{1}} G_{23}^{\rho_{2} \lambda_{2}}\right)\right] \partial_{\rho_{1}} F_{\mu_{1} \nu_{1}} \partial_{\rho_{2}} F_{\mu_{2} \nu_{2}} \partial_{\lambda_{1}} \partial_{\lambda_{2}} F_{\eta \delta}
\end{aligned}
$$

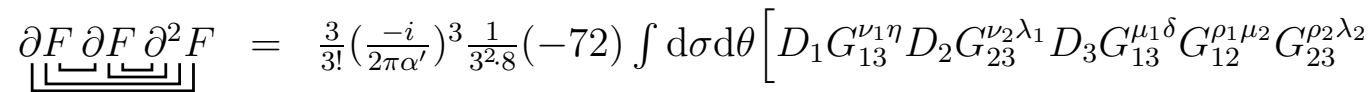

$$
\begin{aligned}
& \left.+D_{1} G_{12}^{\nu_{1} \mu_{2}} D_{2} G_{23}^{\nu_{2} \eta} D_{3} G_{13}^{\mu_{1} \delta} G_{13}^{\rho_{1} \lambda_{1}} G_{23}^{\rho_{2} \lambda_{2}}\right] \partial_{\rho_{1}} F_{\mu_{1} \nu_{1}} \partial_{\rho_{2}} F_{\mu_{2} \nu_{2}} \partial_{\lambda_{1}} \partial_{\lambda_{2}} F_{\eta \delta}
\end{aligned}
$$

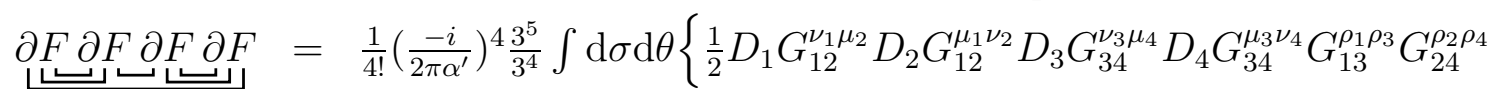

$$
\begin{aligned}
& +D_{1} G_{12}^{\nu_{1} \mu_{2}} D_{2} G_{23}^{\nu_{2} \mu_{3}} D_{3} G_{34}^{\nu_{3} \mu_{4}} D_{4} G_{14}^{\mu_{1} \nu_{4}} G_{12}^{\rho_{1} \rho_{2}} G_{34}^{\rho_{3} \rho_{4}} \\
& +\frac{1}{2}\left[D_{1} G_{12}^{\nu_{1} \mu_{2}} D_{2} G_{12}^{\mu_{1} \nu_{2}} D_{3} G_{13}^{\rho_{1} \nu_{3}} D_{4} G_{34}^{\mu_{3} \nu_{4}} G_{24}^{\rho_{2} \mu_{4}} G_{34}^{\rho_{3} \rho_{4}}+\left(\mu_{4} \leftrightarrow \rho_{4}\right)\right] \\
& \left.+\frac{1}{2}\left[D_{1} G_{12}^{\nu_{1} \mu_{2}} D_{2} G_{12}^{\mu_{1} \nu_{2}} D_{3} G_{13}^{\rho_{1} \nu_{3}} D_{4} G_{34}^{\mu_{3} \nu_{4}} G_{24}^{\rho_{2} \mu_{4}} G_{34}^{\rho_{3} \rho_{4}}+\left(\mu_{3} \leftrightarrow \rho_{3}\right)\right]\right\}
\end{aligned}
$$




$$
\begin{aligned}
& \times \partial_{\rho_{1}} F_{\mu_{1} \nu_{1}} \partial_{\rho_{2}} F_{\mu_{2} \nu_{2}} \partial_{\rho_{3}} F_{\mu_{3} \nu_{3}} \partial_{\rho_{4}} F_{\mu_{4} \nu_{4}}
\end{aligned}
$$

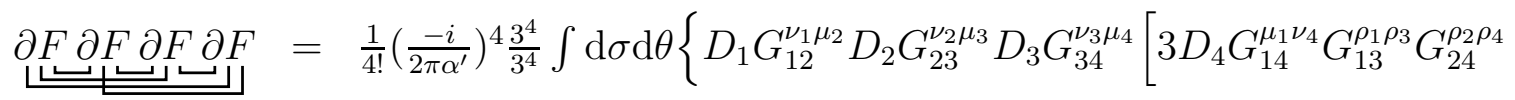

$$
\begin{aligned}
& \left.\left.+2 D_{4} G_{24}^{\rho_{2} \nu_{4}}\left(G_{14}^{\rho_{1} \rho_{4}} G_{23}^{\mu_{1} \rho_{3}}+G_{14}^{\mu_{1} \rho_{4}} G_{23}^{\rho_{1} \rho_{3}}\right)\right]\right\} \\
& \times \partial_{\rho_{1}} F_{\mu_{1} \nu_{1}} \partial_{\rho_{2}} F_{\mu_{2} \nu_{2}} \partial_{\rho_{3}} F_{\mu_{3} \nu_{3}} \partial_{\rho_{4}} F_{\mu_{4} \nu_{4}}
\end{aligned}
$$

In the fomulæ above we have suppressed an overall factor $\langle 0 \mid B(F)\rangle$, which will give an overall factor $-T_{9} \sqrt{\operatorname{det} h}$ in the correction to the action. Also, the contraction symbols are schematical; there is no distinction between lines ending on an $F$ and on a derivative acting on that $F$. The notation ' $D G$ ' means that the contractions are between a $D \phi$ and a $\phi$.

The next step is to perform the integrations over the $\sigma$ 's and $\theta$ 's. We have written the terms in (B.6) so that all $D$ 's act on different $G^{\mu \nu}$ 's. This way of writing the expressions makes it very easy to incorporate the fermionic modes. Since there are as many $D$ 's as there are $\mathrm{d} \theta$ 's in the above expressions and $D_{1} G_{12}^{\mu \nu}=\theta_{1} \partial_{1} D_{12}^{\mu \nu}+\theta_{2} K_{12}^{\mu \nu}$, the integrations over the $\theta$ 's pick out one of the terms (either a $\partial D$ or a $K$ ) in each of the $D G$ 's (the $G$ 's without derivatives get replaced by $D^{\prime}$ 's). Furthermore, since $K_{12}^{\mu \nu}$ and $\partial_{2} D_{12}^{\mu \nu}=-\partial_{1} D_{12}^{\mu \nu}$ are equal after the replacement of integer modes with half-integer ones, the resulting expressions where the integration over the $\theta$ 's picks out some of the $K$ 's instead of the $\partial D$ 's, is obtained by simply changing the relevant summation indices from sums over integers to sums over half integers (sometimes the overall sign also changes). Notice that not all combinations of $\partial D$ 's and $K$ 's are allowed in all instances. The integrations over the $\sigma$ 's will lead to a collection of Kronecker $\delta$ 's (using $\int_{0}^{2 \pi} \mathrm{d} \sigma e^{i n \sigma}=2 \pi \delta_{n, 0}$ ).

The final step is to evaluate the resulting sums and extract the finite parts which will give the contribution to effective action. The power divergent terms cancel between the bosonic and fermionic sectors as a result of supersymmetry. Since the theory is renormalisable the logarithmically divergent terms can be removed by absorbing them in $A_{\mu}$, although we have not verified this fact in detail (the results in [36] should prove to be useful when addressing this question). For the finite terms an additional complication arises: some of these terms are renormalisation scheme dependent, and one would like to get rid of these terms as well. As we have seen in section 2 it is not possible to remove all the renormalisation scheme dependent terms within the scheme used in this paper. Instead another renormalisation scheme has to be used, such as the scheme discussed in [22]. Fortunately, there is a way around this problem as we will see below.

As a sample calculation let us discuss the third type of terms in (B.6). Let us start with the first term. We first note that $\left[D_{3} G_{23}^{\delta \eta}\right]_{2 \rightarrow 3}=\theta_{3}\left(-2 i \alpha^{\prime}\right) h_{A}^{\delta \eta}\left[\sum_{n} e^{-\epsilon n}-\sum_{r} e^{-\epsilon r}\right]$, which, when the regulator is removed, becomes $\theta_{3}\left(-\frac{1}{2}\right)\left(-2 i \alpha^{\prime}\right) h_{A}^{\delta \eta}$. Furthermore, the integrations over $\theta_{1}, \theta_{2}$ picks out the $-\partial_{1} D_{12}^{\nu_{1} \nu_{2}} \partial_{2} D_{23}^{\mu_{2} \lambda_{2}}$ part from $D_{1} G_{12}^{\nu_{1} \nu_{2}} D_{2} G_{23}^{\mu_{2} \lambda_{2}}$. We now note that under a field redefinition, the lowest order Born-Infeld term transforms as $\delta \sqrt{\operatorname{det}(1+F)}=-\frac{1}{2} h^{\mu \nu} \delta F_{\mu \nu}$. The term induced by the field redefinition required to remove the first term in (B.2) thus has exactly the right form to cancel the contribution under discussion. After some relabelling of indices and use of symmetry properties of the propagator, we see that this is indeed the case. Thus, the vanishing of the $\psi_{0}^{2}$ contribution 
to the Wess-Zumino term induces terms which simplify the corrections to the Born-Infeld part as well. For the present term the cancellation is independent of the renormalisation scheme used, but in general the vanishing of the $\psi_{0}^{2}$ contribution to the Wess-Zumino term leads to a strong indication as to which redefinition of $F_{\mu \nu}$ is needed to remove the scheme dependent terms. Some of the terms that are removed in this way involve e.g. $\operatorname{Li}_{2}(-2)$ (see appendix 9 for properties of dilogarithms), which can not be reexpressed in terms of familiar constants.

We now turn to the second term in the third type of terms in (B.6). We get

$$
\begin{gathered}
\frac{3}{3 !}\left(\frac{-i}{2 \pi \alpha^{\prime}}\right)^{3} \frac{1}{3^{2} \cdot 8}(-36)\left(\frac{1}{2}\right) \int \mathrm{d} \sigma \mathrm{d} \theta\left[D_{3} G_{23}^{\delta \eta}\right]_{2 \rightarrow 3} D_{1} G_{12}^{\nu_{1} \mu_{2}} D_{2} G_{12}^{\mu_{1} \nu_{2}} G_{13}^{\rho_{1} \lambda_{1}} G_{23}^{\rho_{2} \lambda_{2}} \\
\times \partial_{\rho_{1}} F_{\mu_{1} \nu_{1}} \partial_{\rho_{2}} F_{\mu_{2} \nu_{2}} \partial_{\lambda_{1}} \partial_{\lambda_{2}} F_{\eta \delta} \\
=\frac{1}{8}\left(\frac{-i}{2 \pi \alpha^{\prime}}\right)^{3} \alpha^{\prime} h_{A}^{\delta \eta}\left(2 \pi \alpha^{\prime}\right)^{3} \alpha^{\prime}\left\{4 h^{\mu_{1} \nu_{2}} h^{\mu_{2} \nu_{1}} h^{\rho_{1} \lambda_{1}} h^{\lambda_{2} \rho_{2}}\left[\sum_{n, p=1}^{\infty} \frac{e^{-\epsilon(3 p+2 n)}}{p^{2}}-(n \leftrightarrow r)\right]\right. \\
\left.\quad-2 h^{\mu_{2} \nu_{1}} h^{\mu_{2} \nu_{1}} h^{\rho_{1} \lambda_{1}} h^{\lambda_{2} \rho_{2}}\left[\sum_{m, n=1}^{\infty} \frac{e^{-2 \epsilon(m+n)}}{(m+n)^{2}}-(n, m \leftrightarrow r, s)\right]\right\} \partial F \partial \partial^{2} F \\
=\alpha^{\prime 2} \frac{\pi^{2}}{12} h_{A}^{\eta \delta} h_{A}^{\mu_{2} \mu_{1}} h^{\nu_{2} \nu_{1}} h^{\rho_{1} \lambda_{1}} h^{\lambda_{2} \rho_{2}} \partial_{\rho_{1}} F_{\mu_{1} \nu_{1}} \partial_{\rho_{2}} F_{\mu_{2} \nu_{2}} \partial_{\lambda_{1}} \partial_{\lambda_{2}} F_{\eta \delta},
\end{gathered}
$$

where we have used results listed in appendix C to evaluate the sums. Again, we have only kept the terms with non-logarithmic finite parts. We see that the above result reproduces the $\partial F \partial F \partial^{2} F$ part of $h^{\rho_{4} \rho_{1}} h^{\rho_{4} \rho_{1}} S_{\rho_{1} \rho_{2}} S_{\rho_{3} \rho_{4}}$ in (4.1).

The story is similar for the other terms in (B.6). The $\partial^{2} F \partial^{2} F$ terms give the corresponding contributions to $h^{\rho_{4} \rho_{1}} h^{\rho_{4} \rho_{1}} S_{\rho_{1} \rho_{2}} S_{\rho_{3} \rho_{4}}$ and $h^{\rho_{4} \rho_{1}} h^{\rho_{4} \rho_{1}} h^{\mu_{4} \mu_{1}} h^{\mu_{4} \mu_{1}} S_{\rho_{1} \rho_{2} \mu_{1} \mu_{2}} S_{\rho_{3} \rho_{4} \mu_{3} \mu_{4}}$. For the case of the remaining type of $\partial F \partial F \partial^{2} F$ terms, the first part is removed by the term induced by the field redefinition needed to cancel $(\mathbb{B . 3})$, whereas the second part gives the $\partial F \partial F \partial^{2} F$ part of $h^{\rho_{4} \rho_{1}} h^{\rho_{4} \rho_{1}} h^{\mu_{4} \mu_{1}} h^{\mu_{4} \mu_{1}} S_{\rho_{1} \rho_{2} \mu_{1} \mu_{2}} S_{\rho_{3} \rho_{4} \mu_{3} \mu_{4}}$. For the first type of $(\partial F)^{4}$ terms in (B.6), the first term gives the corresponding part of $h^{\rho_{4} \rho_{1}} h^{\rho_{4} \rho_{1}} S_{\rho_{1} \rho_{2}} S_{\rho_{3} \rho_{4}}$, whereas the second term gives rise to half of the $(\partial F)^{4}$ part of $h^{\rho_{4} \rho_{1}} h^{\rho_{4} \rho_{1}} h^{\mu_{4} \mu_{1}} h^{\mu_{4} \mu_{1}} S_{\rho_{1} \rho_{2} \mu_{1} \mu_{2}} S_{\rho_{3} \rho_{4} \mu_{3} \mu_{4}}$. The other terms are cancelled by terms induced by field redefinitions needed to remove two-form $(\partial F)^{4}$ contributions to the Wess-Zumino term. Finally, for the second type of $(\partial F)^{4}$ terms, the first term gives rise to the rest of $h^{\rho_{4} \rho_{1}} h^{\rho_{4} \rho_{1}} h^{\mu_{4} \mu_{1}} h^{\mu_{4} \mu_{1}} S_{\rho_{1} \rho_{2} \mu_{1} \mu_{2}} S_{\rho_{3} \rho_{4} \mu_{3} \mu_{4}}$, whereas the other terms are removed by field redefinitions.

In conclusion, we would like to stress that given the large number of contributions involved, the final result is surprisingly simple.

\section{B.3 Four-derivative four- $F$ terms}

In this subsection we will show that when restricting the expression in (4.1) to the terms with at most four F's, we correctly reproduce the previously known result (4.3). Firstly, let us note that any term involving four $\partial$ 's and two $F$ 's can be removed by a field redefinition since by using the Bianchi identity and integrations by parts such a term is necessarily proportional to the lowest order equation of motion, $\partial^{\mu} F_{\mu \nu}=0$. Also, the $F^{4}$ terms induced by the field redefinition removing the $F^{2}$ terms can again be removed. Secondly, any term of the form $F F \partial^{2} F \partial^{2} F$ can be rewritten as $F \partial F \partial F \partial^{2} F$ up to terms 
which are proportional to the lowest order equation of motion, by using the Bianchi identity and integration by parts. To show this fact we note that at least four indices have to be contracted within the $\partial^{2} F \partial^{2} F$ part. Furthermore, these contractions have to be between the two factors since otherwise we get terms proportional to the equation of motion. If one of the contracted indices is on a derivative we integrate by parts with repect to this derivative, which will give us $F \partial F \partial F \partial^{2} F$ terms modulo terms proportional to the equation of motion. The only remaining possibility is $F^{\mu \nu} F^{\eta \delta} \partial_{\mu} \partial_{\eta} F_{\rho \lambda} \partial_{\nu} \partial_{\delta} F^{\rho \lambda}$, which by integration with respect to $\partial_{\mu}$ gives $F \partial F \partial F \partial^{2} F$ terms. Finally, any $(\partial F)^{4}$ can, by integration by parts, be rewritten in terms of $F \partial F \partial F \partial^{2} F$ terms. We thus arrive at the conclusion that any term with four $F$ 's and four $\partial$ 's can (modulo terms proportional to the equations of motion) be rewritten in the form $F \partial F \partial F \partial^{2} F$. By using the Bianchi identity any $F \partial F \partial F \partial^{2} F$ term can be expressed in terms of the following thirteen terms

$$
\begin{aligned}
L_{1} & =F_{\mu \nu} \partial^{\lambda_{1}} F^{\eta \mu} \partial^{\lambda_{2}} F^{\delta \nu} \partial_{\lambda_{1}} \partial_{\lambda_{2}} F_{\eta \delta}, \\
L_{2} & =F_{\mu \nu} \partial^{\eta} F^{\lambda_{1} \mu} \partial^{\lambda_{2}} F^{\delta \nu} \partial_{\lambda_{1}} \partial_{\lambda_{2}} F_{\eta \delta}, \\
L_{3} & =F_{\mu \nu} \partial^{\eta} F^{\lambda_{1} \mu} \partial^{\delta} F^{\lambda_{2} \nu} \partial_{\lambda_{1}} \partial_{\lambda_{2}} F_{\eta \delta}, \\
L_{4} & =F_{\mu \nu} \partial^{\eta} F^{\delta \mu} \partial^{\lambda_{1}} F^{\lambda_{2} \nu} \partial_{\lambda_{1}} \partial_{\lambda_{2}} F_{\eta \delta}, \\
L_{5} & =F_{\mu \nu} \partial_{\rho} F^{\lambda_{1} \mu} \partial^{\eta} F^{\lambda_{2} \rho} \partial_{\lambda_{1}} \partial_{\lambda_{2}} F_{\eta}{ }^{\nu}, \\
L_{6} & =F_{\mu \nu} \partial^{\lambda_{1}} F_{\rho}{ }^{\mu} \partial^{\lambda_{2}} F^{\eta \rho} \partial_{\lambda_{1}} \partial_{\lambda_{2}} F_{\eta}{ }^{\nu}, \\
L_{7} & =F_{\mu \nu} \partial^{\lambda_{1}} F_{\rho}{ }^{\mu} \partial^{\eta} F^{\lambda_{2} \rho} \partial_{\lambda_{1}} \partial_{\lambda_{2}} F_{\eta}{ }^{\nu}, \\
L_{8} & =F_{\mu \nu} \partial_{\rho} F^{\eta \mu} \partial^{\lambda_{1}} F^{\lambda_{2} \rho} \partial_{\lambda_{1}} \partial_{\lambda_{2}} F_{\eta}{ }^{\nu}, \\
L_{9} & =F_{\mu \nu} \partial^{\eta} F_{\rho}{ }^{\mu} \partial^{\lambda_{1}} F^{\lambda_{2} \rho} \partial_{\lambda_{1}} \partial_{\lambda_{2}} F_{\eta}{ }^{\nu}, \\
L_{10} & =F_{\mu \nu} \partial_{\rho} F^{\lambda_{1} \mu} \partial^{\lambda_{2}} F^{\eta \rho} \partial_{\lambda_{1}} \partial_{\lambda_{2}} F_{\eta}{ }^{\nu}, \\
L_{11} & =F_{\mu \nu} \partial_{\rho} F_{\eta}^{\lambda_{1}} \partial^{\rho} F^{\eta \lambda_{2}} \partial_{\lambda_{1}} \partial_{\lambda_{2}} F^{\mu \nu}, \\
L_{12} & =F_{\mu \nu} \partial_{\rho} F_{\eta}^{\lambda_{1}} \partial^{\eta} F^{\rho \lambda_{2}} \partial_{\lambda_{1}} \partial_{\lambda_{2}} F^{\mu \nu}, \\
L_{13} & =F_{\mu \nu} \partial^{\rho} F^{\mu \nu} \partial^{\lambda_{1}} F^{\lambda_{2} \eta} \partial_{\lambda_{1}} \partial_{\lambda_{2}} F_{\eta \rho} .
\end{aligned}
$$

By integrations by parts there will be relations between these terms. There are nine independent such relations, reducing the number of independent elements to four. These can be chosen to be $L_{1}, L_{6}, L_{11}-L_{12}$ and $L_{13}$. By integrations by parts and use of the Bianchi identity these four quantities can in turn (modulo the equation of motion) be expressed in terms of the following ones

$$
\begin{aligned}
& X_{1}=\partial_{\rho} F_{\mu \nu} \partial^{\rho} F^{\nu \mu} \partial_{\lambda} F_{\eta \delta} \partial^{\lambda} F^{\delta \eta}=\operatorname{tr}\left(\partial_{\rho} F \partial^{\rho} F\right) \operatorname{tr}\left(\partial_{\lambda} F \partial^{\lambda} F\right), \\
& X_{2}=\partial_{\rho} F_{\mu \nu} \partial_{\lambda} F^{\nu \mu} \partial^{\rho} F_{\eta \delta} \partial^{\lambda} F^{\delta \eta}=\operatorname{tr}\left(\partial_{\rho} F \partial_{\lambda} F\right) \operatorname{tr}\left(\partial^{\rho} F \partial^{\lambda} F\right), \\
& X_{3}=\partial_{\rho} F_{\mu \nu} \partial^{\rho} F^{\nu \eta} \partial_{\lambda} F_{\eta \delta} \partial^{\lambda} F^{\delta \mu}=\operatorname{tr}\left(\partial_{\rho} F \partial^{\rho} F \partial_{\lambda} F \partial^{\lambda} F\right), \\
& X_{4}=\partial_{\rho} F_{\mu \nu} \partial_{\lambda} F^{\nu \eta} \partial^{\rho} F_{\eta \delta} \partial^{\lambda} F^{\delta \mu}=\operatorname{tr}\left(\partial_{\rho} F \partial_{\lambda} F \partial^{\rho} F \partial^{\lambda} F\right) .
\end{aligned}
$$


For completeness we list the relations between the $X$ 's and the $L$ 's (valid up to terms proportional to the lowest order equation of motion)

$$
\begin{aligned}
L_{1} & =\frac{1}{2} X_{4}-X_{3}, & L_{2} & =\frac{1}{32}\left(-X_{1}+6 X_{2}-24 X_{3}+12 X_{4}\right), \\
L_{3} & =\frac{1}{16}\left(-X_{1}+6 X_{2}-8 X_{3}+4 X_{4}\right), & L_{4} & =\frac{1}{32}\left(-X_{1}+6 X_{2}+8 X_{3}-4 X_{4}\right), \\
L_{5} & =0, & L_{6} & =\frac{1}{2} X_{4}, \\
L_{7} & =\frac{1}{32}\left(-3 X_{1}+10 X_{2}-8 X_{3}+20 X_{4}\right), & L_{8} & =0, \\
L_{9} & =\frac{1}{32}\left(-X_{1}-2 X_{2}+8 X_{3}+12 X_{4}\right), & L_{10} & =\frac{1}{32}\left(X_{1}+2 X_{2}-8 X_{3}+4 X_{4}\right), \\
L_{11} & =\frac{1}{8}\left(X_{1}+2 X_{2}-8 X_{3}+4 X_{4}\right), & L_{12} & =\frac{1}{8}\left(-X_{1}+6 X_{2}-8 X_{3}+4 X_{4}\right), \\
L_{13} & =\frac{1}{4} X_{1} . & &
\end{aligned}
$$

We now have all information needed to check that (4.1) indeed contains the result (4.3). Most of the contributions in (4.1) can be straightforwardly reexpressed in terms of the $X$ 's, whereas some require the use of the above results. We will not give any further details here.

The expression (4.3) can be rewritten in terms of the $t_{8}$ tensor. When this eight-index tensor is contracted with four arbitrary anti-symmetric matrices the result is

$$
\begin{aligned}
t_{8}^{\mu_{1} \cdots \mu_{8}} A_{\mu_{1} \mu_{2}} B_{\mu_{3} \mu_{4}} C_{\mu_{5} \mu_{6}} D_{\mu_{7} \mu_{8}}:= & -2[\operatorname{tr}(A B) \operatorname{tr}(C D)+\operatorname{tr}(A C) \operatorname{tr}(B D)+\operatorname{tr}(A D) \operatorname{tr}(B C)] \\
& +8 \operatorname{tr}(A B C D+A C B D+A D C B)
\end{aligned}
$$

\section{Sums galore}

We here collect some of the sums which appear in the calculations. Some of the expressions involve the dilogarithm, $\operatorname{Li}_{2}(x)$, defined as $\operatorname{Li}_{2}(x)=\sum_{k=1}^{\infty} \frac{x^{k}}{k^{2}}$. Various special values are $\operatorname{Li}_{2}(1)=\frac{\pi^{2}}{6}$ and $\operatorname{Li}_{2}(-1)=-\frac{\pi^{2}}{12}$. The dilogarithm has the following asymptotic expansion for large $x$ : $\operatorname{Li}_{2}(-x) \sim-\frac{1}{2} \ln ^{2}(x)-\frac{\pi^{2}}{6}$. The following relations between $\operatorname{Li}_{2}\left(-\frac{1}{2}\right), \operatorname{Li}_{2}(-2)$ and $\mathrm{Li}_{2}(-3)$ are also useful:

$$
\begin{aligned}
\mathrm{Li}_{2}\left(-\frac{1}{2}\right)+\mathrm{Li}_{2}(-2) & =-\frac{\pi^{2}}{6}, \\
\mathrm{Li}_{2}(-2)+\frac{1}{2} \mathrm{Li}_{2}(-3) & =-\frac{\pi^{2}}{6}-\ln 2 \ln 3 .
\end{aligned}
$$

Further properties and relations can be found in [37]. We now list the sums over integers which are needed in this paper. In the expressions below $a, b \geq 0$ and $\epsilon>0$. The notation $\sim$ should be read 'equal up to $\mathcal{O}(\epsilon)$ corrections'.

$$
\begin{aligned}
& \sum_{n=1}^{\infty} e^{-\epsilon n}=\frac{e^{-\epsilon}}{1-e^{-\epsilon}} \sim \frac{1}{\epsilon}-\frac{1}{2} \\
& \sum_{n=1}^{\infty} \frac{e^{-\epsilon n}}{n}=-\ln \left(1-e^{-\epsilon}\right) \sim-\ln \epsilon
\end{aligned}
$$




$$
\begin{aligned}
& \sum_{m, n=1}^{\infty} \frac{e^{-\epsilon(m+n)}}{m+n}=\ln \left(1-e^{-\epsilon}\right)+\frac{e^{-\epsilon}}{1-e^{-\epsilon}} \sim \frac{1}{\epsilon}+\ln \epsilon-\frac{1}{2} \\
& \sum_{m, n=1}^{\infty} \frac{e^{-\epsilon(m+n)}}{m(m+n)}=\frac{1}{2} \ln ^{2}\left(1-e^{-\epsilon}\right) \sim \frac{1}{2} \ln ^{2} \epsilon \\
& \sum_{m, n=1}^{\infty} \frac{e^{-\epsilon(m+n)}}{(m+n)^{2}}=-\ln \left(1-e^{-\epsilon}\right)-\operatorname{Li}_{2}\left(e^{-\epsilon}\right) \sim-\ln \epsilon+\frac{\pi^{2}}{6} \\
& \sum_{n, m, p, q=1}^{\infty} \frac{e^{-\epsilon(m+n+p+q)}}{m n} \delta(m+p-n-q)=\frac{1}{2} \frac{e^{-2 \epsilon}}{1-e^{-2 \epsilon}}\left[\ln ^{2}\left(1-e^{-2 \epsilon}\right)-2 \operatorname{Li}_{2}\left(-\frac{e^{-2 \epsilon}}{1-e^{-2 \epsilon}}\right)\right] \\
& \sim\left(\frac{1}{2 \epsilon}-\frac{1}{2}\right)\left[\frac{\pi^{2}}{6}+\log \text { terms }\right] \\
& \sum_{m, n=1}^{\infty} \frac{e^{-\epsilon(b(m+n)+a m)}}{m(m+n)}=\ln \left(1-e^{-\epsilon a}\right) \ln \left(e^{-\epsilon a} \frac{1-e^{-\epsilon b}}{1-e^{-\epsilon a}}\right)-\operatorname{Li}_{2}\left(-e^{-\epsilon a} \frac{1-e^{-\epsilon b}}{1-e^{-\epsilon a}}\right) \\
& +\mathrm{Li}_{2}\left(-\frac{e^{-\epsilon a}}{1-e^{-\epsilon a}}\right)-\ln \left(1-e^{-\epsilon a}\right) \ln \left(\frac{e^{-\epsilon a}}{1-e^{-\epsilon a}}\right) \\
& \sim-\mathrm{Li}_{2}\left(-\frac{b}{a}\right)-\frac{\pi^{2}}{6}+\log \text { terms } \\
& \sum_{n, m}^{\infty} \frac{\left.e^{-\epsilon(b(m+n)+a n}\right)}{m(m+n)}=\ln \left(1-e^{-\epsilon b}\right) \ln \left[\frac{1-e^{-\epsilon(b+a)}}{1-e^{-\epsilon a}}\right]+\operatorname{Li}_{2}\left(-e^{-\epsilon a} \frac{1-e^{-\epsilon b}}{1-e^{-\epsilon a}}\right) \\
& -\mathrm{Li}_{2}\left(-\frac{e^{-\epsilon a}}{1-e^{-\epsilon a}}\right) \\
& \sim \operatorname{Li}_{2}\left(-\frac{b}{a}\right)+\frac{\pi^{2}}{6}+\log \text { terms } \\
& \sum_{m, n, k=1}^{\infty} \frac{e^{-\epsilon(b(m+n+k)+a(m+k))}}{m(m+n+k)}=\frac{e^{-\epsilon a}}{1-e^{-\epsilon a}}\left[\ln \left(1-e^{-\epsilon(a+b)}\right) \ln \left(1-e^{-\epsilon a}\right)-\frac{e^{\epsilon a}}{2} \ln ^{2}\left(1-e^{-\epsilon(a+b)}\right)\right. \\
& -\ln \left(1-e^{-\epsilon b}\right) \ln \left(\frac{1-e^{-\epsilon(b+a)}}{1-e^{-\epsilon a}}\right)-\operatorname{Li}_{2}\left(-e^{-\epsilon a} \frac{1-e^{-\epsilon b}}{1-e^{-\epsilon a}}\right) \\
& \left.+\mathrm{Li}_{2}\left(-\frac{e^{-\epsilon a}}{1-e^{-\epsilon a}}\right)\right] \\
& \sim-\left(\frac{1}{a \epsilon}-\frac{1}{2}\right)\left[\operatorname{Li}_{2}\left(-\frac{b}{a}\right)+\frac{\pi^{2}}{6}\right]+\log \text { terms } \\
& \sum_{m, n, k=1}^{\infty} \frac{e^{-\epsilon(b(m+n+k)+a k)}}{m(m+n+k)}=\frac{1}{2} \frac{1}{1-e^{-a \epsilon}}\left\{\ln \left(1-e^{-\epsilon b}\right)\left[e^{-\epsilon a} \ln \left(1-e^{-\epsilon b}\right)-2 \ln \left(\frac{1-e^{-\epsilon(a+b)}}{1-e^{-\epsilon a}}\right)\right]\right. \\
& \left.-2 \operatorname{Li}_{2}\left(-e^{-\epsilon a} \frac{1-e^{-\epsilon b}}{1-e^{-\epsilon a}}\right)+2 \operatorname{Li}_{2}\left(-\frac{e^{-\epsilon a}}{1-e^{-\epsilon a}}\right)\right\} \\
& \sim-\left(\frac{1}{a \epsilon}+\frac{1}{2}\right)\left[\operatorname{Li}_{2}\left(-\frac{b}{a}\right)+\frac{\pi^{2}}{6}\right]+\log \text { terms } \\
& \sum_{m, n, k=1}^{\infty} \frac{e^{-\epsilon(b(m+n+k)+a m)}}{(m+n)(m+n+k)}=\frac{1}{1-e^{-a \epsilon}}\left[-\ln \left(1-e^{-\epsilon(a+b)}\right) \ln \left(1-e^{-\epsilon a}\right)+\frac{e^{-\epsilon a}}{2} \ln ^{2}\left(1-e^{-\epsilon a}\right)\right. \\
& +\ln \left(1-e^{-\epsilon b}\right) \ln \left(\frac{1-e^{-\epsilon(b+a)}}{1-e^{-\epsilon a}}\right)+\operatorname{Li}_{2}\left(-e^{-\epsilon a} \frac{1-e^{-\epsilon b}}{1-e^{-\epsilon a}}\right) \\
& \left.-\mathrm{Li}_{2}\left(-\frac{e^{-\epsilon a}}{1-e^{-\epsilon a}}\right)\right] \\
& \sim\left(\frac{1}{a \epsilon}+\frac{1}{2}\right)\left[\operatorname{Li}_{2}\left(-\frac{b}{a}\right)+\frac{\pi^{2}}{6}\right]+\log \text { terms }
\end{aligned}
$$




$$
\begin{aligned}
\sum_{m, n, k=1}^{\infty} \frac{e^{-\epsilon(4 m+2 k+2 n)}}{(m+n)(m+k)}= & \frac{2}{1-e^{-\epsilon}} \ln \left(1+e^{-\epsilon}\right)-\ln ^{2}\left(1-e^{-\epsilon}\right)+\ln \left(1-e^{-\epsilon}\right) \\
& -\ln \left(1+e^{-\epsilon}\right) \sim \log \text { terms } .
\end{aligned}
$$

The method we used to derive these expressions was by first differentiating with respect to either $\epsilon, a$ or $b$ in such a way as to obtain a simpler sum. Integration then gives the original sum. The integration constant can be fixed using the fact that the sum goes to zero when the parameter goes to infinity. The sums over half-integer modes needed in this paper can be simply related to the above sums by replacing all half-integer modes by $n-\frac{1}{2}$, with $n \in \mathbb{Z}_{+}$, and possibly also shifting some of the summation variables. Examples include

$$
\begin{aligned}
\sum_{r=\frac{1}{2}}^{\infty} e^{-\epsilon r} & =e^{\frac{\epsilon}{2}} \sum_{n=1}^{\infty} e^{-\epsilon n} \\
\sum_{r, s=\frac{1}{2} ; k=1}^{\infty} \frac{e^{-\epsilon(b(r+s+k)+a r)}}{(r+s)(r+s+k)} & =e^{\frac{\epsilon a}{2}}\left[\sum_{m, n, k=1}^{\infty} \frac{e^{-\epsilon(b(m+n+k)+a m)}}{(m+n)(m+n+k)}+\sum_{m, k=1}^{\infty} \frac{e^{-\epsilon(b(m+k)+a m)}}{m(m+k)}\right] .
\end{aligned}
$$

We also need the following sums whose complete expressions are rather involved; we therefore only list the relevant terms

$$
\begin{aligned}
& \sum_{n, m, k=1}^{\infty} \frac{e^{-\epsilon(4 m+3 n+3 k)}}{(m+k)(m+n)}-\sum_{r, s, t=\frac{1}{2}}^{\infty} \frac{e^{-\epsilon(4 r+3 s+3 t)}}{(r+s)(r+t)} \sim \operatorname{Li}_{2}(-3)+\frac{1}{2} \frac{\pi^{2}}{6}+\log \text { terms } \\
& \sum_{n, m, k=1}^{\infty} \frac{e^{-\epsilon(3 m+2 n+2 k)}}{(m+k)(m+n)}-\sum_{r, s, t=\frac{1}{2}}^{\infty} \frac{e^{-\epsilon(4 r+3 s+3 t)}}{(r+s)(r+t)} \sim \operatorname{Li}_{2}(-2)+\frac{1}{2} \frac{\pi^{2}}{6}+\log \text { terms } .
\end{aligned}
$$

\section{References}

[1] M. R. Douglas, "Branes within branes." hep-th/9512077; M. Li, "Boundary States of D-Branes and Dy-Strings." Nucl. Phys. B460 (1996) 351, hep-th/9510161.

[2] E. S. Fradkin and A. A. Tseytlin, "Nonlinear electrodynamics from quantized strings." Phys. Lett. B163 (1985) 123.

[3] A. Abouelsaood, C. G. Callan, C. R. Nappi, and S. A. Yost, "Open strings in background gauge fields." Nucl. Phys. B280 (1987) 599; E. Bergshoeff, E. Sezgin, C. N. Pope, and P. K. Townsend, "The Born-Infeld action from conformal invariance of the open superstring." Phys. Lett. 188B (1987) 70.

[4] R. G. Leigh, "Dirac-Born-Infeld action from Dirichlet sigma model." Mod. Phys. Lett. A4 (1989) 2767. 
[5] M. Cederwall, A. von Gussich, B. E. W. Nilsson, and A. Westerberg, "The Dirichlet super three-brane in ten-dimensional type IIB supergravity." Nucl. Phys. B490 (1997) 163, hep-th/9610148; M. Aganagic, C. Popescu, and J. H. Schwarz, "D-brane actions with local kappa symmetry." Phys. Lett. B393 (1997) 311, hep-th/9610249; M. Cederwall, A. von Gussich, B. E. W. Nilsson, P. Sundell, and A. Westerberg, "The Dirichlet super $p$-branes in ten-dimensional type IIA and IIB supergravity." Nucl. Phys. B490 (1997) 179, hep-th/9611159; E. Bergshoeff and P. K. Townsend, "Super D-branes." Nucl. Phys. B490 (1997) 145, hep-th/9611173; M. Aganagic, C. Popescu, and J. H. Schwarz, "Gauge invariant and gauge fixed D-brane actions." Nucl. Phys. B495 (1997) 99, hep-th/9612080.

[6] E. Alvarez, J. L. F. Barbón, and J. Borlaf, "T-duality for open strings." Nucl. Phys. B479 (1996) 218, hep-th/9603089; E. Bergshoeff and M. De Roo, "D-branes and T-duality." Phys. Lett. B380 (1996) 265, hep-th/9603123.

[7] M. B. Green, C. M. Hull, and P. K. Townsend, "D-brane Wess-Zumino actions, T duality and the cosmological constant." Phys. Lett. B382 (1996) 65, hep-th/9604119.

[8] M. R. Garousi and R. C. Myers, "World-volume interactions on D-branes." Nucl. Phys. B542 (1999) 73, hep-th/9809100.

[9] M. Cederwall and A. Westerberg, "World-volume fields, $\operatorname{SL}(2, \mathbb{Z})$ and duality: the type IIB 3-brane," JHEP 9802, 004 (1998), hep-th/9710007; P. S. Howe, O. Raetzel, and E. Sezgin, "On brane actions and superembeddings." JHEP 08 (1998) 011, hep-th/9804051; A. Westerberg and N. Wyllard, "Supersymmetric brane actions from interpolating dualisations." Nucl. Phys. B560 (1999) 683, hep-th/9904117.

[10] C. Bachas, "D-brane dynamics." Phys. Lett. B374 (1996) 37, hep-th/9511043.

[11] N. Seiberg and E. Witten, "String theory and noncommutative geometry." JHEP 09 (1999) 032, hep-th/9908142.

[12] G. W. Gibbons and D. A. Rasheed, "Electric-magnetic duality rotations in nonlinear electrodynamics." Nucl. Phys. B454 (1995) 185, hep-th/9506035; M. B. Green and M. Gutperle, "Comments on three-branes." Phys. Lett. B377 (1996) 28, hep-th/9602077.

[13] A. A. Tseytlin, "Selfduality of Born-Infeld action and Dirichlet three-brane of type IIB superstring theory." Nucl. Phys. B469 (1996) 51, hep-th/9602064.

[14] S. Deser, J. G. McCarthy, and Ö. Sariog̃lu, "Good propagation' and duality invariance constraints on scalar, gauge vector and gravity actions." Class. Quant. Grav. 16 (1999) 841, hep-th/9809153. 
[15] M. B. Green, J. A. Harvey, and G. Moore, "I-brane inflow and anomalous couplings on D-branes." Class. Quant. Grav. 14 (1997) 47, hep-th/9605033;

Y.-K. E. Cheung and Z. Yin, "Anomalies, branes, and currents." Nucl. Phys. B517 (1998) 69, hep-th/9710206.

[16] J. Polchinski, "TASI lectures on D-branes." hep-th/9611050;

C. V. Johnson, "D-brane primer." hep-th/0007170.

[17] C. P. Bachas, P. Bain, and M. B. Green, "Curvature terms in D-brane actions and their M-theory origin." JHEP 05 (1999) 011, hep-th/9903210.

[18] M. R. Garousi and R. C. Myers, "Superstring Scattering from D-Branes." Nucl. Phys. B475 (1996) 193, hep-th/9603194; A. Hashimoto and I. R. Klebanov, "Scattering of strings from D-branes." Nucl. Phys. Proc. Suppl. 55B (1997) 118, hep-th/9611214.

[19] B. Craps and F. Roose, "Anomalous D-brane and orientifold couplings from the boundary state." Phys. Lett. B445 (1998) 150, hep-th/9808074; J. F. Morales, C. A. Scrucca, and M. Serone, "Anomalous couplings for D-branes and O-planes." Nucl. Phys. B552 (1999) 291, hep-th/9812071; B. Stefanski, Jr., "Gravitational couplings of D-branes and O-planes." Nucl. Phys. B548 (1999) 275, hep-th/9812088.

[20] A. Neveu and J. Scherk, "Connection between Yang-Mills fields and dual models." Nucl. Phys. B36 (1972) 155; J. Scherk and J. H. Schwarz, "Dual models for non-hadrons." Nucl. Phys. B81 (1974) 118; T. Yoneya, "Connection of dual models to electrodynamics and gravidynamics." Prog. Theor. Phys. 51 (1974) 1907.

[21] A. A. Tseytlin, "Vector field effective action in the open superstring theory." Nucl. Phys. B276 (1986) 391, erratum ibid. B291 (1987) 876; A. A. Tseytlin, "Ambiguity in the effective action in string theories." Phys. Lett. B176 (1986) 92; A. A. Tseytlin, "Renormalisation of Möbius infinities and partition function representation for string theory effective action." Phys. Lett. B202 (1988) 81.

[22] O. D. Andreev and A. A. Tseytlin, "Partition function representation for the open superstring effective action: cancellation of Möbius infinities and derivative corrections to Born-Infeld Lagrangian.” Nucl. Phys. B311 (1988) 205.

[23] C. G. Callan, C. Lovelace, C. R. Nappi, and S. A. Yost, "String loop corrections to beta functions." Nucl. Phys. B288 (1987) 525; C. G. Callan, C. Lovelace, C. R. Nappi, and S. A. Yost, "Adding holes and crosscaps to the superstring." Nucl. Phys. B293 (1987) 83.

[24] C. G. Callan, C. Lovelace, C. R. Nappi, and S. A. Yost, "Loop corrections to superstring equations of motion." Nucl. Phys. B308 (1988) 221. 
[25] M. B. Green and P. Wai, "The Insertion of boundaries in world sheets." Nucl. Phys. B431 (1994) 131; M. B. Green and M. Gutperle, "Light-cone supersymmetry and D-branes." Nucl. Phys. B476 (1996) 484, hep-th/9604091.

[26] P. Di Vecchia and A. Liccardo, "D branes in string theory, I." hep-th/9912161; P. Di Vecchia and A. Liccardo, "D branes in string theory, II." hep-th/9912275.

[27] K. Hashimoto, "Corrections to D-brane action and generalized boundary state." Phys. Rev. D61 (2000) 106002, hep-th/9909027.

[28] K. Hashimoto, "Generalized supersymmetric boundary state." JHEP 04 (2000) 023, hep-th/9909095.

[29] J. H. Schwarz, "Superstring theory.” Phys. Rept. 89 (1982) 223.

[30] P. Di Vecchia, M. Frau, A. Lerda, and A. Liccardo, “(F,D $p)$ bound states from the boundary state." Nucl. Phys. B565 (2000) 397, hep-th/9906214.

[31] P. Haggi-Mani, U. Lindström, and M. Zabzine, "Boundary conditions, supersymmetry and $A$-field coupling for an open string in a $B$-field background." hep-th/0004061.

[32] N. Wyllard, work in progress.

[33] M. B. Green and M. Gutperle, "D-instanton induced interactions on a D3-brane." hep-th/0002011.

[34] T. Damour, S. Deser, and J. G. McCarthy, "Nonsymmetric gravity theories: inconsistencies and a cure." Phys. Rev. D47 (1993) 1541, gr-qc/9207003.

[35] M. Abou-Zeid and C. M. Hull, "Intrinsic geometry of D-branes." Phys. Lett. B404 (1997) 264, hep-th/9704021; M. Abou-Zeid, "Actions for curved branes." hep-th/0001127.

[36] O. D. Andreev and A. A. Tseytlin, "Two loop beta function in the open string sigma model and equivalence with string effective equations of motion." Mod. Phys. Lett. A3 (1988) 1349.

[37] L. Lewin, Polylogarithms and associated functions. North-Holland, 1981. 